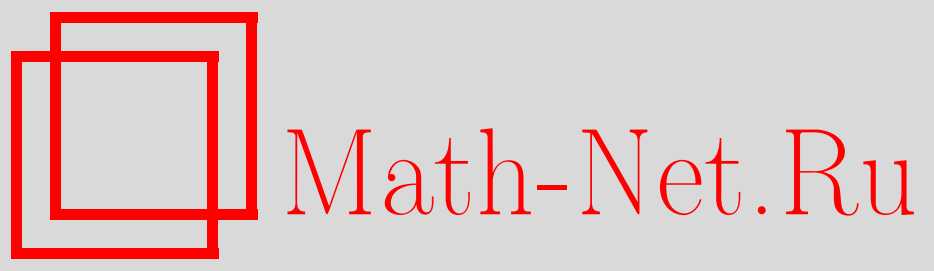

Ю. М. Зиновьев, Лоренц-ковариантные обобщенные функции со спектральными условиями, ТМФ, 2008, том 156, номер 3, 328-350

DOI: https://doi.org/10.4213/tmf6251

Использование Общероссийского математического портала Math-Net.Ru подразумевает, что вы прочитали и согласны с пользовательским соглашением http://www.mathnet.ru/rus/agreement

Параметры загрузки:

IP: 35.173 .219 .12

26 апреля 2023 г., 13:03:07

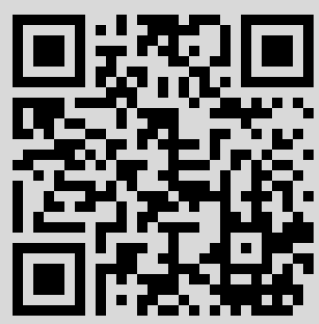




\section{ЛОРЕНЦ-КОВАРИАНТНЫЕ ОБОБЩЕННЫЕ ФУНКЦИИ СО СПЕКТРАЛЬНЫМИ УСЛОВИЯМИ}

Получено описание лоренц-ковариантных обобщенных функций умеренного роста с носителями в произведении замкнутых верхних световых конусов.

Ключевые слова: лоренц-ковариантные обобщенные функции.

\section{1. ВВЕДЕНИЕ}

Физические воззрения на квантовые поля обычно формулируются в виде системы аксиом [1]-[4]. Вакуумные ожидания произведений квантовых полей являются преобразованиями Фурье лоренц-ковариантных обобщенных функций умеренного роста с носителями в произведении замкнутых верхних световых конусов [2]-[4]. Лоренц-инвариантные обобщенные функции одной переменной $x \in \mathbb{R}^{4}$ изучались в работах [5], [6]. Описание лоренц-инвариантных обобщенных функций умеренного роста с носителями, лишь по одному аргументу лежащими в гиперболоиде $\left\{x \in \mathbb{R}^{4}: x^{0}>0,(x, x) \geqslant \mu>0\right\}$, было получено в работе [7]. Авторы работ [5]-[7] стремились получить описание лоренц-инвариантных обобщенных функций в терминах обобщенных функций, заданных на пространстве орбит группы Лоренца. Это пространство орбит имеет сложную структуру. В работе [8] замечено, что обобщенная функция умеренного роста с носителем в замкнутом верхнем световом конусе может быть представлена как результат применения некоторой степени волнового оператора к дифференцируемой функции с носителем в замкнутом верхнем световом конусе. Для описания лоренц-ковариантных дифференцируемых функций граница замкнутого верхнего светового конуса не важна. Мера этой границы равна нулю.

В этой работе мы получим описание лоренц-ковариантных обобщенных функций умеренного роста с носителями в произведении замкнутых верхних световых конусов. Таким образом будет получено описание вакуумных ожиданий от произведений квантовых полей, удовлетворяющих свойству лоренц-ковариантности и спектральному условию [2]-[4].

* Математический институт им. В. А. Стеклова РАН, Москва, Россия. E-mail: zinoviev@mi.ras.ru 
Мы изучим также специальный класс лоренц-ковариантных обобщенных функций. Эти обобщенные функции являются суммами обобщенных функций, похожих на произведения одномерных лоренц-ковариантных обобщенных функций.

\section{2. ЛОРЕНЦ-КОВАРИАНТНОСТЬ И СПЕКТРАЛЬНОЕ УСЛОВИЕ}

Определим для комплексной $(2 \times 2)$-матрицы

$$
A=\left(\begin{array}{ll}
A_{11} & A_{12} \\
A_{21} & A_{22}
\end{array}\right)
$$

следующие $(2 \times 2)$-матрицы:

$$
A^{\mathrm{T}}=\left(\begin{array}{ll}
A_{11} & A_{21} \\
A_{12} & A_{22}
\end{array}\right), \quad \bar{A}=\left(\begin{array}{ll}
\bar{A}_{11} & \bar{A}_{12} \\
\bar{A}_{21} & \bar{A}_{22}
\end{array}\right), \quad A^{*}=(\bar{A})^{\mathrm{T}} .
$$

Матрица (2.1) называется эрмитовой, если $A^{*}=A$. Выберем следующий базис в пространстве эрмитовых $(2 \times 2)$-матриц:

$$
\sigma^{0}=\left(\begin{array}{ll}
1 & 0 \\
0 & 1
\end{array}\right), \quad \sigma^{1}=\left(\begin{array}{cc}
0 & 1 \\
1 & 0
\end{array}\right), \quad \sigma^{2}=\left(\begin{array}{cc}
0 & -i \\
i & 0
\end{array}\right), \quad \sigma^{3}=\left(\begin{array}{cc}
1 & 0 \\
0 & -1
\end{array}\right) .
$$

Сопоставим вектору $x \in \mathbb{R}^{4}$ эрмитову матрицу

$$
\tilde{x}=\sum_{\mu=0}^{3} x^{\mu} \sigma^{\mu}
$$

и введем билинейную форму

$$
(x, y)=x^{0} y^{0}-\sum_{k=1}^{3} x^{k} y^{k}
$$

при этом $(x, x)$ - метрика Минковского.

Группа $S L(2, \mathbb{C})$ состоит из комплексных $(2 \times 2)$-матриц $(2.1)$ с определителями, равными единице. Группа $S U(2)$ является максимальной компактной подгруппой группы $S L(2, \mathbb{C})$. Она образована матрицами из группы $S L(2, \mathbb{C})$, удовлетворяющими уравнению $A A^{*}=\sigma^{0}$. Опишем неприводимые представления группы $S U(2)$. Рассмотрим полуцелые числа $l \in \mathbb{Z}_{+} / 2$, т.е. $l=0,1 / 2,1,3 / 2, \ldots$. Определим представление группы $S U(2)$ на пространстве многочленов степени не выше $2 l$ :

$$
T_{l}(A) \phi(z)=\left(A_{12} z+A_{22}\right)^{2 l} \phi\left(\frac{A_{11} z+A_{21}}{A_{12} z+A_{22}}\right) .
$$

Рассмотрим полуцелые числа $n=-l,-l+1, \ldots, l-1, l$ и выберем базис многочленов, состоящий из функций

$$
\psi_{n}(z)=\frac{1}{\sqrt{(l-n) !(l+n) !}} z^{l-n}
$$


Из определений (2.6), (2.7) следует

$$
T_{l}(A) \psi_{n}(z)=\sum_{m=-l}^{l} \psi_{m}(z) t_{m n}^{l}(A),
$$

где

$$
\begin{aligned}
t_{m n}^{l}(A)= & \sqrt{(l-m) !(l+m) !(l-n) !(l+n) !} \times \\
& \times \sum_{j=-\infty}^{\infty} \frac{A_{11}^{l-m-j} A_{12}^{j} A_{21}^{m-n+j} A_{22}^{l+n-j}}{\Gamma(j+1) \Gamma(l-m-j+1) \Gamma(m-n+j+1) \Gamma(l+n-j+1)}
\end{aligned}
$$

и $\Gamma(z)$ - гамма-функция. Функция $(\Gamma(z))^{-1}$ равна нулю для $z=0,-1,-2, \ldots$, следовательно, ряд (2.8) является многочленом.

Соотношение (2.6) определяет представление группы $S U(2)$. Таким образом, многочлен (2.8) определяет представление группы $S U(2)$

$$
t_{m n}^{l}(A B)=\sum_{k=-l}^{l} t_{m k}^{l}(A) t_{k n}^{l}(B) .
$$

Это $(2 l+1)$-мерное неприводимое представление (см. книгу [9], главу III, раздел 2.3).

Соотношения (2.8), (2.9) имеют аналитическое продолжение на матрицы из группы $S L(2, \mathbb{C})$. Делая замену $j \rightarrow j+n-m$ переменной суммирования в равенстве $(2.8)$, получаем

$$
t_{m n}^{l}(A)=t_{n m}^{l}\left(A^{\mathrm{T}}\right) .
$$

Согласно [9] (см. главу III, раздел 8.3) для любой матрицы $A \in S U(2)$ имеем

$$
\begin{aligned}
& t_{m_{1} n_{1}}^{l_{1}}(A) t_{m_{2} n_{2}}^{l_{2}}(A)= \\
& \quad=\sum_{l_{3} \in \mathbb{Z}_{+} / 2} \sum_{m_{3}, n_{3}=-l_{3}}^{l_{3}} C\left(l_{1}, l_{2}, l_{3} ; m_{1}, m_{2}, m_{3}\right) C\left(l_{1}, l_{2}, l_{3} ; n_{1}, n_{2}, n_{3}\right) t_{m_{3} n_{3}}^{l_{3}}(A) .
\end{aligned}
$$

Коэффициент Клебша-Гордана $C\left(l_{1}, l_{2}, l_{3} ; m_{1}, m_{2}, m_{3}\right)$ отличен от нуля лишь для $m_{3}=m_{1}+m_{2}$ и полуцелых чисел $l_{1}, l_{2}, l_{3} \in \mathbb{Z}_{+} / 2$, удовлетворяющих условию треугольника: можно построить треугольник со сторонами длины $l_{1}, l_{2}, l_{3}$ и с целым периметром $l_{1}+l_{2}+l_{3}$. Это означает, что $l_{3}$ является одним из полуцелых чисел $\left|l_{1}-l_{2}\right|,\left|l_{1}-l_{2}\right|+1, \ldots, l_{1}+l_{2}-1, l_{1}+l_{2}$. Пусть числа $l_{1}, l_{2}, l_{3} \in \mathbb{Z}_{+} / 2$ удовлетворяют условию треугольника, а $m_{i}=-l_{i},-l_{i}+1, \ldots, l_{i}-1, l_{i}, i=1,2,3, m_{3}=m_{1}+m_{2}$. Тогда согласно [9] (см. главу III, раздел 8.3)

$$
\begin{aligned}
C\left(l_{1},\right. & \left.l_{2}, l_{3} ; m_{1}, m_{2}, m_{3}\right)=(-1)^{l_{1}-l_{3}+m_{2}}\left(2 l_{3}+1\right)^{1 / 2} \times \\
& \times\left(\frac{\left(l_{1}+l_{2}-l_{3}\right) !\left(l_{1}+l_{3}-l_{2}\right) !\left(l_{2}+l_{3}-l_{1}\right) !\left(l_{3}-m_{3}\right) !\left(l_{3}+m_{3}\right) !}{\left(l_{1}+l_{2}+l_{3}+1\right) !\left(l_{1}-m_{1}\right) !\left(l_{1}+m_{1}\right) !\left(l_{2}-m_{2}\right) !\left(l_{2}+m_{2}\right) !}\right)^{1 / 2} \times \\
& \times \sum_{j=0}^{l_{2}+l_{3}-l_{1}} \frac{(-1)^{j}\left(l_{1}+m_{1}+j\right) !\left(l_{2}+l_{3}-m_{1}-j\right) !}{j ! \Gamma\left(l_{3}-m_{3}-j+1\right) \Gamma\left(l_{1}-l_{2}+m_{3}+j+1\right)\left(l_{2}+l_{3}-l_{1}-j\right) !} .
\end{aligned}
$$


Пусть $d A$ - нормированная мера Хаара на группе $S U(2)$. Согласно [9] (глава III, раздел 8.3)

$$
\begin{aligned}
& C\left(l_{1}, l_{2}, l_{3} ; m_{1}, m_{2}, m_{3}\right) C\left(l_{1}, l_{2}, l_{3} ; n_{1}, n_{2}, n_{3}\right)= \\
& \quad=\left(2 l_{3}+1\right) \int_{S U(2)} d A t_{m_{1} n_{1}}^{l_{1}}(A) t_{m_{2} n_{2}}^{l_{2}}(A) \overline{t_{m_{3} n_{3}}^{l_{3}}(A)} .
\end{aligned}
$$

Коэффициенты многочлена (2.8) вещественны. С помощью соотношений (2.10) и равенства $A^{*}=A^{-1}$ перепишем равенство (2.13) в виде

$$
\begin{aligned}
& C\left(l_{1}, l_{2}, l_{3} ; m_{1}, m_{2}, m_{3}\right) C\left(l_{1}, l_{2}, l_{3} ; n_{1}, n_{2}, n_{3}\right)= \\
& \quad=\left(2 l_{3}+1\right) \int_{S U(2)} d A t_{m_{1} n_{1}}^{l_{1}}(A) t_{m_{2} n_{2}}^{l_{2}}(A) t_{n_{3} m_{3}}^{l_{3}}\left(A^{-1}\right) .
\end{aligned}
$$

Если полуцелые числа $l_{1}, l_{2}, l_{3} \in \mathbb{Z}_{+} / 2$ удовлетворяют условию треугольника, то согласно [9] (глава III, раздел 8.3)

$$
C\left(l_{1}, l_{2}, l_{3} ; l_{1},-l_{2}, l_{1}-l_{2}\right)=\left(\frac{\left(2 l_{3}+1\right)\left(2 l_{1}\right) !\left(2 l_{2}\right) !}{\left(l_{1}+l_{2}-l_{3}\right) !\left(l_{1}+l_{2}+l_{3}+1\right) !}\right)^{1 / 2}
$$

Выберем в равенстве $(2.14) n_{1}=l_{1}, n_{2}=-l_{2}$. Тогда из соотношений $(2.9),(2.14)$ и инвариантности меры Хаара $d A$ следует, что

$$
\begin{aligned}
& C\left(l_{1}, l_{2}, l_{3} ; l_{1},-l_{2}, l_{1}-l_{2}\right) \sum_{n_{1}=-l_{1}}^{l_{1}} \sum_{n_{2}=-l_{2}}^{l_{2}} t_{m_{1} n_{1}}^{l_{1}}(A) t_{m_{2} n_{2}}^{l_{2}}(A) C\left(l_{1}, l_{2}, l_{3} ; n_{1}, n_{2}, m_{3}\right)= \\
&=\left(2 l_{3}+1\right) \int_{S U(2)} d B\left(\sum_{n_{1}=-l_{1}}^{l_{1}} t_{m_{1} n_{1}}^{l_{1}}(A) t_{n_{1} l_{1}}^{l_{1}}(B)\right) \times \\
& \quad \times\left(\sum_{n_{2}=-l_{2}}^{l_{2}} t_{m_{2} n_{2}}^{l_{2}}(A) t_{n_{2},-l_{2}}^{l_{2}}(B)\right) t_{l_{1}-l_{2}, m_{3}}^{l_{3}}\left(B^{-1}\right)= \\
&=\left(2 l_{3}+1\right) \int_{S U(2)} d B t_{m_{1} l_{1}}^{l_{1}}(A B) t_{m_{2},-l_{2}}^{l_{2}}(A B) t_{l_{1}-l_{2}, m_{3}}^{l_{3}}\left(B^{-1}\right)= \\
&=\left(2 l_{3}+1\right) \int_{S U(2)} d B t_{m_{1} l_{1}}^{l_{1}}(B) t_{m_{2},-l_{2}}^{l_{2}}(B) t_{l_{1}-l_{2}, m_{3}}^{l_{3}}\left(B^{-1} A\right)= \\
&=\sum_{n_{3}=-l_{3}}^{l_{3}}\left(2 l_{3}+1\right) \int_{S U(2)} d B t_{m_{1} l_{1}}^{l_{1}}(B) t_{m_{2},-l_{2}}^{l_{2}}(B) t_{l_{1}-l_{2}, n_{3}}^{l_{3}}\left(B^{-1}\right) t_{n_{3} m_{3}}^{l_{3}}(A)= \\
&= C\left(l_{1}, l_{2}, l_{3} ; l_{1},-l_{2}, l_{1}-l_{2}\right) \sum_{n_{3}=-l_{3}}^{l_{3}} C\left(l_{1}, l_{2}, l_{3} ; m_{1}, m_{2}, n_{3}\right) t_{n_{3} m_{3}}^{l_{3}}(A) .
\end{aligned}
$$

Из соотношений $(2.15),(2.16)$ следует равенство

$$
\begin{gathered}
\sum_{n_{1}=-l_{1}}^{l_{1}} \sum_{n_{2}=-l_{2}}^{l_{2}} t_{m_{1} n_{1}}^{l_{1}}(A) t_{m_{2} n_{2}}^{l_{2}}(A) C\left(l_{1}, l_{2}, l_{3} ; n_{1}, n_{2}, m_{3}\right)= \\
\quad=\sum_{n_{3}=-l_{3}}^{l_{3}} C\left(l_{1}, l_{2}, l_{3} ; m_{1}, m_{2}, n_{3}\right) t_{n_{3} m_{3}}^{l_{3}}(A),
\end{gathered}
$$


которое можно аналитически продолжить на любую матрицу из группы $S L(2, \mathbb{C})$.

Рассмотрим для натуральных чисел $m, n$ и полуцелых чисел $l_{1}, \ldots, l_{n+1}, i_{1}, \ldots$ $\ldots, \dot{l}_{n+1} \in \mathbb{Z}_{+} / 2, m_{i}=-l_{i},-l_{i}+1, \ldots, l_{i}-1, l_{i}, \dot{m}_{i}=-\dot{l}_{i},-\dot{l}_{i}+1, \ldots, \dot{l}_{i}-1, \dot{l}_{i}, i=$ $1, \ldots, n+1$, семейство обобщенных функций

$$
F_{m_{1}, \ldots, m_{n+1} ; \dot{m}_{1}, \ldots, \dot{m}_{n+1}}^{l_{1}, \ldots, l_{n+1} ; \dot{l}_{1}, \ldots, i_{n+1}}\left(x_{1}, \ldots, x_{m}\right) \in S^{\prime}\left(\mathbb{R}^{4 m}\right) .
$$

Это семейство называется лоренц-ковариантной обобщенной функцией, если для любой матрицы $A \in S L(2, \mathbb{C})$

$$
\begin{array}{r}
F_{m_{1}, \ldots, m_{n+1} ; \dot{m}_{1}, \ldots, \dot{m}_{n+1}}^{l_{1}, \ldots, l_{n+1} ; i_{1}, \ldots, i_{n+1}}\left(A \tilde{x}_{1} A^{*}, \ldots, A \tilde{x}_{m} A^{*}\right)=\sum_{k_{1}=-l_{1}}^{l_{1}} \ldots \sum_{k_{n+1}=-l_{n+1}}^{l_{n+1}} \sum_{\dot{k}_{1}=-\dot{l}_{1}}^{i_{1}} \ldots \\
\ldots \sum_{\dot{k}_{n+1}=-i_{n+1}}^{i_{n+1}}\left(\prod_{i=1}^{n+1} t_{m_{i} k_{i}}^{l_{i}}(A) t_{\dot{m}_{i} \dot{k}_{i}}^{i_{i}}(\bar{A})\right) F_{k_{1}, \ldots, k_{n+1} ; k_{1}, \ldots, \dot{k}_{n+1}}^{l_{1}, \ldots, l_{n+1} ; i_{1}, \ldots, i_{n+1}}\left(\tilde{x}_{1}, \ldots, \tilde{x}_{m}\right),
\end{array}
$$

где $(2 \times 2)$-матрица $\tilde{x}$ задана соотношением $(2.4)$. Полуцелые числа $l_{1}, \ldots, l_{n+1}$, $i_{1}, \ldots, i_{n+1}$ в $(2.18)$ не произвольны. Выберем в равенстве $(2.18)$ матрицу $A=-\sigma^{0}$. Для этой матрицы

$$
A \tilde{x}_{j} A^{*}=\tilde{x}_{j}, \quad j=1, \ldots, m .
$$

Из определения (2.8) следует, что

$$
t_{m_{j} k_{j}}^{l_{j}}\left(-\sigma^{0}\right)=(-1)^{2 l_{j}} \delta_{m_{j} k_{j}} .
$$

Следовательно, равенство (2.18) справедливо для матрицы $A=-\sigma^{0}$, если

$$
(-1)^{2\left(l_{1}+\cdots+l_{n+1}+i_{1}+\cdots+i_{n+1}\right)}=1,
$$

т.е. сумма $l_{1}+\cdots+l_{n+1}+i_{1}+\cdots+i_{n+1}$ является целым числом. Далее мы предполагаем, что это условие всегда выполнено.

Следуя работе [8], мы получим представление для обобщенной функции умеренного роста с носителем в замкнутом верхнем световом конусе

$$
\bar{V}_{+}=\left\{x \in \mathbb{R}^{4}: x^{0} \geqslant 0,(x, x) \geqslant 0\right\} .
$$

ЛЕмма 1. Пусть обобщенная функиия $F(x) \in S^{\prime}\left(\mathbb{R}^{4}\right)$ имеет носитель в замкнутом верхнем световом конусе. Тогда существует такое натуральное число $q$, что

$$
\begin{gathered}
F(x)=\left(\partial_{x}, \partial_{x}\right)^{q} f(x) \\
\left(\partial_{x}, \partial_{x}\right)=\left(\frac{\partial}{\partial x^{0}}\right)^{2}-\sum_{k=1}^{3}\left(\frac{\partial}{\partial x^{k}}\right)^{2},
\end{gathered}
$$

где дифферениируемая функиия $f(x)$ с носителем в замкнутом верхнем световом конусе полиномиально ограничена. 
ДокАзАтельство. Введем функцию-ступеньку

$$
\theta(x)= \begin{cases}1, & x \geqslant 0 \\ 0, & x<0 .\end{cases}
$$

Согласно [10] (см. раздел 30, формулы (126), (146))

$$
\frac{1}{8 \pi}\left(\partial_{x}, \partial_{x}\right)^{2}\left(\theta\left(x^{0}\right) \theta((x, x))\right)=\delta(x) .
$$

Отсюда следует, что для любого натурального числа $q \geqslant 2$

$$
\frac{1}{2 \pi 4^{q-1}(q-2) !(q-1) !}\left(\partial_{x}, \partial_{x}\right)^{q}\left((x, x)^{q-2} \theta\left(x^{0}\right) \theta((x, x))\right)=\delta(x) .
$$

Для любого вектора $x \in \bar{V}_{+}$пересечение носителя функции

$$
(x-y, x-y)^{q-2} \theta\left(x^{0}-y^{0}\right) \theta((x-y, x-y))
$$

как функции переменной $y$ с конусом $\bar{V}_{+}$компактно. Эта функция $2(q-3)$ раз дифференцируема. Пусть обобщенная функция $F(x) \in S^{\prime}\left(\mathbb{R}^{4}\right)$ имеет носитель в конусе $\bar{V}_{+}$. Тогда для достаточно большого натурального числа $q$ функция

$$
f(x)=\frac{1}{2 \pi 4^{q-1}(q-2) !(q-1) !} \int d^{4} y F(y)(x-y, x-y)^{q-2} \theta\left(x^{0}-y^{0}\right) \theta((x-y, x-y))
$$

дифференцируема и имеет носитель в конусе $\bar{V}_{+}$. Эта функция полиномиально ограничена.

Рассмотрим соотношение (2.18) для простейшего случая $m=n=1$.

ПредложениЕ. Любая обобщенная функиия $F_{m_{1}, m_{2} ; \dot{m}_{1}, \dot{m}_{2}}^{l_{1}, l_{2} ; l_{1}, i_{2}}(x) \in S^{\prime}\left(\mathbb{R}^{4}\right)$ с носителем в замкнутом верхнем световом конусе, удовлетворяющая соотношению ковариантности (2.18) для $m=n=1$, имеет следующий вид:

$$
\begin{aligned}
& \int d^{4} x F_{m_{1}, m_{2} ; \dot{m}_{1}, \dot{m}_{2}}^{l_{1}, l_{2} ; i_{1}, i_{2}}(x) \phi(x)= \\
&= \sum_{l_{3} \in \mathbb{Z}_{+} / 2} \sum_{m_{3}, \dot{m}_{3}=-l_{3}}^{l_{3}} C\left(l_{1}, l_{2}, l_{3} ; m_{1}, m_{2}, m_{3}\right) C\left(\dot{l}_{1}, \dot{l}_{2}, l_{3} ; \dot{m}_{1}, \dot{m}_{2}, \dot{m}_{3}\right) \times \\
& \quad \times \int d^{4} x \theta\left(x^{0}\right) \theta((x, x)) t_{m_{3} \dot{m}_{3}}^{l_{3}}(\tilde{x}) f^{l_{1}, l_{2}, l_{3} ; i_{1}, i_{2}}(\sqrt{(x, x)})\left(\partial_{x}, \partial_{x}\right)^{q} \phi(x),
\end{aligned}
$$

где $\phi(x) \in S\left(\mathbb{R}^{4}\right), q$ - натуралъное число, коэфбициент $C\left(l_{1}, l_{2}, l_{3} ; m_{1}, m_{2}, m_{3}\right)$ задан соотношением (2.12), $(2 \times 2)$-матрица $\tilde{x}$ - соотношением $(2.4)$, многочлен $t_{m n}^{l}(A)$ соотношением (2.8), дифференцируемая функиия $f^{l_{1}, l_{2}, l_{3} ; i_{1}, i_{2}}(s)$ с носителем на положительной полуоси полиномиально ограничена.

ДокАЗАТЕЛЬСтво. Коэффициент $C\left(l_{1}, l_{2}, l_{3} ; m_{1}, m_{2}, m_{3}\right)$ не равен нулю лишь для чисел $l_{1}, l_{2}, l_{3} \in \mathbb{Z}_{+} / 2$, удовлетворяющих условию треугольника. Следовательно, суммы в правой части равенства (2.25) конечны. 
Функция $f^{l_{1}, l_{2}, l_{3} ; i_{1}, i_{2}}(s)$ полиномиально ограничена, следовательно, интегралы в правой части равенства (2.25) сходятся абсолютно. Правая часть равенства (2.25) определяет обобщенную функцию из $S^{\prime}\left(\mathbb{R}^{4}\right)$.

Из формул $(2.10),(2.17)$ следует, что правая часть равенства (2.25) удовлетворяет соотношению ковариантности (2.18) для $m=n=1$.

Докажем, что любая обобщенная функция из $S^{\prime}\left(\mathbb{R}^{4}\right)$ с носителем в конусе $\bar{V}_{+}$, удовлетворяющая соотношению ковариантности (2.18) для $m=n=1$, имеет вид (2.25).

Если носитель обобщенной функции $F_{m_{1}, m_{2} ; \dot{m}_{1}, \dot{m}_{2}}^{l_{1}, l_{2} ; i_{1}, i_{2}}(x) \in S^{\prime}\left(\mathbb{R}^{4}\right)$ лежит в конусе $\bar{V}_{+}$, то справедливо представление (2.19), где дифференцируемая функция $f_{m_{1}, m_{2} ; \dot{m}_{1}, \dot{m}_{2}}^{l_{1}, l_{2} ; i_{1}, i_{2}}(x)$ задана равенством $(2.24)$.

Введем для открытого верхнего светового конуса

$$
V_{+}=\left\{x \in \mathbb{R}^{4}: x^{0}>0,(x, x)>0\right\}
$$

координаты

$$
\tilde{x}=\mu g(t, z) g(t, z)^{*}
$$

где $\mu$ - положительное число, $t$ - положительное число, $z$ - комплексное число, а

$$
g(t, z)=\left(\begin{array}{cc}
t^{-1} & 0 \\
z & t
\end{array}\right)
$$

Равенство (2.26) является следствием разложения Гаусса для эрмитовой положительно определенной $(2 \times 2)$-матрицы.

Введем функцию

$$
\begin{aligned}
& f_{m_{1}, m_{2} ; \dot{m}_{1}, \dot{m}_{2}}^{l_{1}, l_{2} ; i_{1}, \dot{l}_{2}}(\mu, g(t, z))= \\
& =\sum_{k_{1}=-l_{1}}^{l_{1}} \sum_{k_{2}=-l_{2}}^{l_{2}} \sum_{\dot{k}_{1}=-i_{1}}^{i_{1}} \sum_{\dot{k}_{2}=-i_{2}}^{i_{2}}\left(\prod_{i=1}^{2} t_{m_{i} k_{i}}^{l_{i}}\left(g(t, z)^{-1}\right) \overline{t_{\dot{m}_{i} \dot{k}_{i}}^{i_{i}}\left(g(t, z)^{-1}\right)}\right) \times \\
& \quad \times f_{k_{1}, k_{2} ; \dot{k}_{1}, \dot{k}_{2}}^{l_{1}, l_{2} ; i_{1}, \dot{l}_{2}} \\
& \quad\left(\mu g(t, z) g(t, z)^{*}\right) .
\end{aligned}
$$

Функция $f_{m_{1}, m_{2} ; \dot{m}_{1}, \dot{m}_{2}}^{l_{1}, l_{2} ; i_{1}, i_{2}}(\tilde{x})$, определенная равенством $(2.24)$, удовлетворяет соотношению ковариантности $(2.18)$ для $m=n=1$. Следовательно, для любой матрицы $A$ типа (2.27)

$$
f_{m_{1}, m_{2} ; \dot{m}_{1}, \dot{m}_{2}}^{l_{1}, l_{2} ; i_{1}, i_{2}}(\mu, A g(t, z))=f_{m_{1}, m_{2} ; \dot{m}_{1}, \dot{m}_{2}}^{l_{1}, l_{2} ; i_{1}, i_{2}}(\mu, g(t, z)) .
$$

Выберем базис алгебры Ли группы матриц (2.27):

$$
a_{1}=\left(\begin{array}{ll}
0 & 0 \\
1 & 0
\end{array}\right), \quad a_{2}=\left(\begin{array}{cc}
0 & 0 \\
i & 0
\end{array}\right), \quad a_{3}=\frac{1}{2}\left(\begin{array}{cc}
1 & 0 \\
0 & -1
\end{array}\right)
$$


Из равенства (2.29) следуют три соотношения:

$$
\begin{aligned}
& \left.\frac{\partial}{\partial s} f_{m_{1}, m_{2} ; \dot{m}_{1}, \dot{m}_{2}}^{l_{1}, l_{2} ; i_{1}, i_{2}}\left(\mu, e^{s a_{1}} g(t, z)\right)\right|_{s=0}=\frac{1}{t} \frac{\partial}{\partial \operatorname{Re} z} f_{m_{1}, m_{2} ; \dot{m}_{1}, \dot{m}_{2}}^{l_{1}, l_{2} ; i_{1}, i_{2}}(\mu, g(t, z))=0 \\
& \left.\frac{\partial}{\partial s} f_{m_{1}, m_{2} ; \dot{m}_{1}, \dot{m}_{2}}^{l_{1}, l_{2} ; l_{1}, \dot{l}_{2}}\left(\mu, e^{s a_{2}} g(t, z)\right)\right|_{s=0}=\frac{1}{t} \frac{\partial}{\partial \operatorname{Im} z} f_{m_{1}, m_{2} ; \dot{m}_{1}, \dot{m}_{2}}^{l_{1}, l_{2} ; i_{1}, i_{2}}(\mu, g(t, z))=0 \\
& \left.\frac{\partial}{\partial s} f_{m_{1}, m_{2} ; \dot{m}_{1}, \dot{m}_{2}}^{l_{1}, l_{2} ; l_{1}, \dot{l}_{2}}\left(\mu, e^{s a_{3}} g(t, z)\right)\right|_{s=0}= \\
& =-\frac{1}{2}\left(\operatorname{Re} z \frac{\partial}{\partial \operatorname{Re} z}+\operatorname{Im} z \frac{\partial}{\partial \operatorname{Im} z}+t \frac{\partial}{\partial t}\right) f_{m_{1}, m_{2} ; \dot{m}_{1}, \dot{m}_{2}}^{l_{1}, l_{2} ; l_{1}, \dot{l}_{2}}(\mu, g(t, z))=0
\end{aligned}
$$

из которых вытекает, что функция (2.28) не зависит от переменной $g(t, z)$. Поэтому из определения (2.28) имеем

$$
f_{m_{1}, m_{2} ; \dot{m}_{1}, \dot{m}_{2}}^{l_{1}, l_{2} ; i_{1}, i_{2}}(\mu, g(t, z))=f_{m_{1}, m_{2} ; \dot{m}_{1}, \dot{m}_{2}}^{l_{1}, l_{2} ; i_{1}, i_{2}}\left(\mu, \sigma^{0}\right)=f_{m_{1}, m_{2} ; \dot{m}_{1}, \dot{m}_{2}}^{l_{1}, l_{2} ; i_{1}, i_{2}}\left(\mu \sigma^{0}\right) .
$$

Из соотношений $(2.9),(2.28)$ и (2.31) получаем

$$
\begin{aligned}
& f_{m_{1}, m_{2} ; \dot{m}_{1}, \dot{m}_{2}}^{l_{1}, l_{2}, i_{1}, i_{2}}\left(\mu g(t, z) g(t, z)^{*}\right)= \\
& =\sum_{k_{1}=-l_{1}}^{l_{1}} \sum_{k_{2}=-l_{2}}^{l_{2}} \sum_{\dot{k}_{1}=-\dot{l}_{1}}^{i_{1}} \sum_{\dot{k}_{2}=-\dot{l}_{2}}^{i_{2}}\left(\prod_{i=1}^{2} t_{m_{i} k_{i}}^{l_{i}}(g(t, z)) \overline{t_{\dot{m}_{i} \dot{k}_{i}}^{\dot{l}_{i}}(g(t, z))}\right) \times \\
& \quad \times f_{k_{1}, l_{2} ; i_{2} ; \dot{k}_{1}, \dot{k}_{2}}^{l_{1}, \dot{k}_{2}}\left(\mu \sigma^{0}\right) .
\end{aligned}
$$

Выберем в (2.24) такое натуральное число $q$, что функция $f_{m_{1}, m_{2} ; \dot{m}_{1}, \dot{m}_{2}}^{l_{1}, l_{2} ; i_{1}, i_{2}}(\tilde{x})$ дифференцируема $l_{1}+l_{2}+i_{1}+i_{2}+1$ раз (соотношение ковариантности (2.18) предполагает, что $l_{1}+l_{2}+\dot{l}_{1}+\dot{l}_{2}$ является целым неотрицательным числом). Носитель этой функции лежит в конусе $\bar{V}_{+}$. Следовательно, ее первые $l_{1}+l_{2}+i_{1}+i_{2}$ производных равны нулю на границе конуса $\bar{V}_{+}$, поэтому функция $f_{k_{1}, k_{2} ; \dot{k}_{1}, \dot{k}_{2}}^{l_{1}, l_{2} \dot{l}_{1}, i_{2}}\left(\mu \sigma^{0}\right)$ дифференцируема $l_{1}+l_{2}+i_{1}+i_{2}+1$ раз, и ее первые $l_{1}+l_{2}+\dot{l}_{1}+\dot{l}_{2}$ производных равны нулю в точке $\mu=0$. В силу (2.24) функция $f_{k_{1}, k_{2} ; \dot{k}_{1}, \dot{k}_{2}}^{l_{1}, l_{2} ; \dot{l}_{1}, \dot{l}_{2}}\left(\mu \sigma^{0}\right)$ полиномиально ограничена.

Делая замену переменных (2.26), мы с помощью (2.32) получаем

$$
\begin{aligned}
& \int d^{4} x f_{m_{1}, m_{2} ; \dot{m}_{1}, \dot{m}_{2}}^{l_{1} l_{2} ; \dot{l}_{1}, i_{2}}(\tilde{x}) \phi(\tilde{x})= \\
& =\sum_{k_{1}=-l_{1}}^{l_{1}} \sum_{k_{2}=-l_{2}}^{l_{2}} \sum_{k_{1}=-i_{1}}^{i_{1}} \sum_{k_{2}=-i_{2}}^{i_{2}} \int_{0}^{\infty} \mu^{3} d \mu \int_{0}^{\infty} t^{-3} d t \int d \operatorname{Re} z \int d \operatorname{Im} z \times \\
& \times\left(\prod_{i=1}^{2} t_{m_{i} k_{i}}^{l_{i}}(g(t, z)) \overline{t_{\dot{m}_{i} \dot{k}_{i}}^{\dot{l}_{i}}(g(t, z))}\right) 2 f_{k_{1}, k_{2} ; \dot{k}_{1}, \dot{k}_{2}}^{l_{1}, l_{2} ; \dot{l}_{1}, \dot{l}_{2}}\left(\mu \sigma^{0}\right) \phi\left(\mu g(t, z) g(t, z)^{*}\right) \text {. }
\end{aligned}
$$

Функция $f_{m_{1}, m_{2} ; \dot{m}_{1}, \dot{m}_{2}}^{l_{1}, l_{2} ; l_{1}, i_{2}}(\tilde{x})$, определенная равенством $(2.24)$, удовлетворяет соотношению ковариантности (2.18) для $m=n=1$. Для любой матрицы $A \in S U(2)$ 
справедливо равенство $A \sigma^{0} A^{*}=\sigma^{0}$. Тогда из соотношения ковариантности $(2.18)$ для матрицы $A \in S U(2)$ следует

$f_{m_{1}, m_{2} ; \dot{m}_{1}, \dot{m}_{2}}^{l_{1}, l_{2} ; \dot{l}_{1}, \dot{l}_{2}}\left(\mu \sigma^{0}\right)=\sum_{k_{1}=-l_{1}}^{l_{1}} \sum_{k_{2}=-l_{2}}^{l_{2}} \sum_{\dot{k}_{1}=-\dot{l}_{1}}^{i_{1}} \sum_{\dot{k}_{2}=-\dot{l}_{2}}^{i_{2}}\left(\prod_{i=1}^{2} t_{m_{i} k_{i}}^{l_{i}}(A) \overline{t_{\dot{m}_{i} \dot{k}_{i}}^{i_{i}}(A)}\right) f_{k_{1}, k_{2} ; \dot{k}_{1}, \dot{k}_{2}}^{l_{1}, l_{2} ; \dot{l}_{1}, i_{2}}\left(\mu \sigma^{0}\right)$.

Проинтегрируем это равенство с нормированной мерой Хаара $d A$ на группе $S U(2)$. Из формул (2.11), (2.13) получаем

$$
\begin{aligned}
& f_{m_{1}, m_{2} ; \dot{m}_{1}, \dot{m}_{2}}^{l_{1}, l_{2} ; i_{1}, i_{2}}\left(\mu \sigma^{0}\right)=\sum_{l_{3} \in \mathbb{Z}_{+} / 2} \sum_{m_{3}, k_{3}=-l_{3}}^{l_{3}} \sum_{k_{1}=-l_{1}}^{l_{1}} \sum_{k_{2}=-l_{2}}^{l_{2}} \times \\
& \quad \times \sum_{\dot{k}_{1}=-\dot{l}_{1}}^{i_{1}} \sum_{\dot{k}_{2}=-\dot{l}_{2}}^{i_{2}} C\left(l_{1}, l_{2}, l_{3} ; m_{1}, m_{2}, m_{3}\right) C\left(\dot{l}_{1}, \dot{l}_{2}, l_{3} ; \dot{m}_{1}, \dot{m}_{2}, m_{3}\right) \times \\
& \quad \times \frac{1}{2 l_{3}+1} C\left(l_{1}, l_{2}, l_{3} ; k_{1}, k_{2}, k_{3}\right) C\left(\dot{l}_{1}, \dot{l}_{2}, l_{3} ; \dot{k}_{1}, \dot{k}_{2}, k_{3}\right) f_{k_{1}, k_{2} ; \dot{k}_{1}, \dot{k}_{2}}^{l_{1}, l_{2} ; \dot{l}_{1}, i_{2}}\left(\mu \sigma^{0}\right) .
\end{aligned}
$$

Введем функцию

$$
\begin{aligned}
& f^{l_{1}, l_{2}, l_{3} ; i_{1}, i_{2}}(\mu)=\sum_{k_{1}=-l_{1}}^{l_{1}} \sum_{k_{2}=-l_{2}}^{l_{2}} \sum_{\dot{k}_{1}=-\dot{l}_{1}}^{i_{1}} \sum_{\dot{k}_{2}=-\dot{l}_{2}}^{i_{2}} \sum_{k_{3}=-l_{3}}^{l_{3}} \frac{1}{2 l_{3}+1} \times \\
& \times C\left(l_{1}, l_{2}, l_{3} ; k_{1}, k_{2}, k_{3}\right) C\left(\dot{l}_{1}, \dot{l}_{2}, l_{3} ; \dot{k}_{1}, \dot{k}_{2}, k_{3}\right) \mu^{-2 l_{3}} f_{k_{1}, k_{2} ; k_{1}, \dot{k}_{2}}^{l_{1}, l_{2} ; i_{1}, i_{2}}\left(\mu \sigma^{0}\right) .
\end{aligned}
$$

Функция $f_{m_{1}, m_{2} ; \dot{m}_{1}, \dot{m}_{2}}^{l_{1}, l_{2} ; i_{1}, i_{2}}\left(\mu \sigma^{0}\right)$ дифференцируема $l_{1}+l_{2}+i_{1}+i_{2}+1$ раз, и ее первые $l_{1}+l_{2}+\dot{l}_{1}+\dot{l}_{2}$ производных равны нулю в точке $\mu=0$, следовательно, функция (2.36) дифференцируема. Функция $f_{m_{1}, m_{2} ; \dot{m}_{1}, \dot{m}_{2}}^{l_{1}, l_{2} ; i_{1}, i_{2}}\left(\mu \sigma^{0}\right)$ полиномиально ограничена, следовательно, функция (2.36) полиномиально ограничена.

Используя соотношения $(2.35),(2.36)$ и формулы $(2.10),(2.17)$ для матрицы $(2.27)$, перепишем равенство (2.33) в следующем виде:

$$
\begin{aligned}
& \int d^{4} x f_{m_{1}, m_{2} ; \dot{m}_{1}, \dot{m}_{2}}^{l_{1}, l_{2} ; i_{1}, i_{2}}(\tilde{x}) \phi(\tilde{x})= \\
&= \sum_{l_{3} \in \mathbb{Z}_{+} / 2} \sum_{m_{3}, \dot{m}_{3}=-l_{3}}^{l_{3}} \int_{0}^{\infty} \mu^{3} d \mu \int_{0}^{\infty} t^{-3} d t \int d \operatorname{Re} z \int d \operatorname{Im} z \times \\
& \quad \times C\left(l_{1}, l_{2}, l_{3} ; m_{1}, m_{2}, m_{3}\right) C\left(\dot{l}_{1}, \dot{l}_{2}, l_{3} ; \dot{m}_{1}, \dot{m}_{2}, \dot{m}_{3}\right) \times \\
& \quad \times t_{m_{3} \dot{m}_{3}}^{l_{3}}\left(\mu g(t, z) g(t, z)^{*}\right) 2 f^{l_{1}, l_{2}, l_{3} ; \dot{l}_{1}, i_{2}}(\mu) \phi\left(\mu g(t, z) g(t, z)^{*}\right) .
\end{aligned}
$$

Делая замену переменных, обратную замене переменных (2.26), мы с помощью равенства (2.19) получаем (2.25).

Рассмотрим обобщенную функцию умеренного роста с носителем в конусе $\bar{V}_{+}$, удовлетворяющую соотношению ковариантности (2.18) для $m=1, n=0$. Этот случай соответствует равенству (2.25) с $l_{2}=\dot{l}_{2}=0, m_{2}=\dot{m}_{2}=0$. Из (2.12) имеем

$$
C\left(l_{1}, 0, l_{3} ; m_{1}, 0, m_{3}\right)=\delta_{l_{1} l_{3}} \delta_{m_{1} m_{3}} .
$$


Группу матриц вида $(2.27)$ обозначим $Z_{-} D(2)$. Для матрицы из группы $S L(2, \mathbb{C})$ справедливо разложение Грама

$$
\begin{gathered}
\left(\begin{array}{ll}
A_{11} & A_{12} \\
A_{21} & A_{22}
\end{array}\right)=\left(\begin{array}{cc}
t^{-1} & 0 \\
z & t
\end{array}\right)\left(\begin{array}{cc}
\alpha & \beta \\
-\bar{\beta} & \bar{\alpha}
\end{array}\right), \\
t=\frac{1}{\sqrt{\left|A_{11}\right|^{2}+\left|A_{12}\right|^{2}}}, \quad z=\frac{\bar{A}_{11} A_{21}+\bar{A}_{12} A_{22}}{\sqrt{\left|A_{11}\right|^{2}+\left|A_{12}\right|^{2}}}, \\
\alpha=\frac{A_{11}}{\sqrt{\left|A_{11}\right|^{2}+\left|A_{12}\right|^{2}}}, \quad \beta=\frac{A_{12}}{\sqrt{\left|A_{11}\right|^{2}+\left|A_{12}\right|^{2}}} .
\end{gathered}
$$

Первая матрица в правой части (2.39) есть матрица $g(t, z) \in Z_{-} D(2)$, вторая матрица есть матрица $u \in S U(2)$. Пусть $d u$ - нормированная мера Хаара на группе $S U(2)$. Рассмотрим меру

$$
d(g(t, z) u)=t^{-3} d t d \operatorname{Re} z d \operatorname{Im} z d u
$$

на группе $S L(2, \mathbb{C})$. Нетрудно проверить, что мера $d g(t, z)=t^{-3} d t d \operatorname{Re} z d \operatorname{Im} z$ инвариантна по отношению к левым сдвигам на группе $Z_{-} D(2)$, следовательно, мера (2.40) инвариантна по отношению к левому умножению матрицы (2.39) на любую матрицу из группы $Z_{-} D(2)$. В силу разложения Грама для доказательства инвариантности меры (2.40) по отношению к левому умножению матрицы (2.39) на любую матрицу из группы $S L(2, \mathbb{C})$ достаточно доказать инвариантность меры $(2.40)$ по отношению к левому умножению матрицы (2.39) на любую матрицу из группы $S U(2)$. Пусть комплексные числа $\alpha, \beta$ удовлетворяют соотношению $|\alpha|^{2}+|\beta|^{2}=1$. Из разложения Грама (2.39) следует,что

$$
\begin{gathered}
\left(\begin{array}{cc}
\alpha & \beta \\
-\bar{\beta} & \bar{\alpha}
\end{array}\right)\left(\begin{array}{cc}
t^{-1} & 0 \\
z & t
\end{array}\right)=\left(\begin{array}{cc}
t_{1}^{-1} & 0 \\
z_{1} & t_{1}
\end{array}\right)\left(\begin{array}{cc}
\alpha_{1} & \beta_{1} \\
-\bar{\beta}_{1} & \bar{\alpha}_{1}
\end{array}\right) \\
t_{1}=\frac{1}{\sqrt{\left|\alpha t^{-1}+\beta z\right|^{2}+|\beta|^{2} t^{2}}}, \quad z_{1}=\frac{\left(\bar{\alpha} t^{-1}+\bar{\beta} \bar{z}\right)\left(-\bar{\beta} t^{-1}+\bar{\alpha} z\right)+\bar{\alpha} \bar{\beta} t^{2}}{\sqrt{\left|\alpha t^{-1}+\beta z\right|^{2}+|\beta|^{2} t^{2}}}, \\
\alpha_{1}=\frac{\alpha t^{-1}+\beta z}{\sqrt{\left|\alpha t^{-1}+\beta z\right|^{2}+|\beta|^{2} t^{2}}}, \quad \beta_{1}=\frac{\beta t}{\sqrt{\left|\alpha t^{-1}+\beta z\right|^{2}+|\beta|^{2} t^{2}}} .
\end{gathered}
$$

Мера Хаара $d u$ инвариантна по отношению к сдвигам на группе $S U(2)$. Для доказательства инвариантности меры (2.40) по отношению к левому умножению матрицы (2.39) на любую матрицу из группы $S U(2)$ достаточно доказать равенство

$$
t_{1}^{-3} d t_{1} d \operatorname{Re} z_{1} d \operatorname{Im} z_{1}=t^{-3} d t d \operatorname{Re} z d \operatorname{Im} z,
$$

где числа $t_{1}, z_{1}$ заданы после $(2.41)$. Введем переменные $x^{1}, x^{2}, x^{3}$ такие, что

$$
\sqrt{|\mathbf{x}|^{2}+1} \sigma^{0}+\sum_{k=1}^{3} x^{k} \sigma^{k}=g(t, z) g(t, z)^{*}, \quad|\mathbf{x}|^{2}=\sum_{k=1}^{3}\left(x^{k}\right)^{2} .
$$

Нетрудно показать, что

$$
\frac{1}{2 \sqrt{|\mathbf{x}|^{2}+1}} d x^{1} d x^{2} d x^{3}=t^{-3} d t d \operatorname{Re} z d \operatorname{Im} z .
$$

2 Теоретическая и математическая физика, т. 156, № 3, 2008 г. 
Отображение $g(t, z) \rightarrow g\left(t_{1}, z_{1}\right)$ соответствует вращению

$$
\left(\begin{array}{cc}
\alpha & \beta \\
-\bar{\beta} & \bar{\alpha}
\end{array}\right)\left(\sqrt{|\mathbf{x}|^{2}+1} \sigma^{0}+\sum_{k=1}^{3} x^{k} \sigma^{k}\right)\left(\begin{array}{cc}
\alpha & \beta \\
-\bar{\beta} & \bar{\alpha}
\end{array}\right)^{*}
$$

вектора $\mathbf{x} \in \mathbb{R}^{3}$, определенного в (2.43). Из инвариантности меры (2.44) по отношению к вращениям следует равенство (2.42). Таким образом, мера (2.40) инвариантна по отношению к левым сдвигам на группе $S L(2, \mathbb{C})$.

ТЕОРема 1. Любая обобщенная функиия

$$
F_{m_{1}, \ldots, m_{n+1} ; \dot{m}_{1}, \ldots, \dot{m}_{n+1}}^{l_{1}, \ldots, l_{n+1} ; i_{1}, \ldots, i_{n+1}}\left(x_{1}, \ldots, x_{m}\right) \in S^{\prime}\left(\mathbb{R}^{4 m}\right)
$$

с носителем в произведении $\bar{V}_{+}^{\times m}$ конусов, удовлетворяющая соотношению ковариантности (2.18), имеет следующий вид:

$$
\begin{array}{rl}
\int d^{4 m} & x F_{m_{1}, \ldots, m_{n+1} ; \dot{m}_{1}, \ldots, \dot{m}_{n+1}}^{l_{1}, \ldots, l_{n+1} ; i_{1}, \ldots, i_{n+1}}\left(x_{1}, \ldots, x_{m}\right) \phi\left(x_{1}, \ldots, x_{m}\right)= \\
= & \sum_{k_{1}=-l_{1}}^{l_{1}} \ldots \sum_{k_{n+1}=-l_{n+1}}^{l_{n+1}} \sum_{\dot{k}_{1}=-i_{1}}^{i_{1}} \ldots \sum_{\dot{k}_{n+1}=-i_{n+1}}^{i_{n+1}} \int_{0}^{\infty} \mu^{4 m-1} d \mu \int_{0}^{\infty} t^{-3} d t \times \\
& \times \int d \operatorname{Re} z \int d \operatorname{Im} z \int_{S U(2)} d A \int d^{4 m} p \times \\
& \times \delta\left(\sum_{j=1}^{m} \tilde{p}_{j}-\sigma^{0}\right)\left(\prod_{i=1}^{n+1} t_{m_{i} k_{i}}^{l_{i}}(g(t, z) A) \overline{t_{\dot{m}_{i} \dot{k}_{i}}}(g(t, z) A)\right) \times \\
& \times 2 f_{k_{1}, \ldots, k_{n+1} ; \dot{k}_{1}, \ldots, \dot{k}_{n+1}}^{l_{1}, \ldots, l_{n+1} ; i_{1}, \ldots, i_{n+1}}\left(\mu p_{1}, \ldots, \mu p_{m}\right) \times \\
& \times\left.\left(\prod_{i=1}^{m}\left(\partial_{x_{i}}, \partial_{x_{i}}\right)^{q}\right) \phi\left(x_{1}, \ldots, x_{m}\right)\right|_{\tilde{x}_{i}=\mu g(t, z) A \tilde{p}_{i}(g(t, z) A)^{*}, i=1, \ldots, m}
\end{array}
$$

где $\phi\left(x_{1}, \ldots, x_{m}\right) \in S\left(\mathbb{R}^{4 m}\right), d A$ - нормированная мера Хаара на группе $S U(2)$, $(2 \times 2)$-матрица $g(t, z)$ имеет вид $(2.27)$, многочлен $t_{m n}^{l}(A)$ определен в $(2.8), q-$ натуральное число, функиия $f_{m_{1}, \ldots, m_{n+1} ; \dot{m}_{1}, \ldots, \dot{m}_{n+1}}^{l_{1}, \ldots, l_{n+1} ; l_{1}, \ldots, i_{n+1}}\left(p_{1}, \ldots, p_{m}\right)$ полиномиально ограничена и дифберениируема $l_{1}+\cdots+l_{n+1}+\dot{l}_{1}+\cdots+\dot{l}_{n+1}+1$ раз (соотношение ковариантности (2.18) предполагает, что $l_{1}+\cdots+l_{n+1}+i_{1}+\cdots+i_{n+1}$ является неотрицательным чельм числом), носитель функиии $f_{m_{1}, \ldots, m_{n+1} ; \dot{m}_{1}, \ldots, \dot{m}_{n+1}}^{l_{1}, \ldots, l_{n+1} ; i_{1}, \ldots, i_{n+1}}\left(p_{1}, \ldots, p_{m}\right)$ лежит в произведении $\bar{V}_{+}^{\times m}$ конусов.

ДокАЗАТЕЛЬство. Мера (2.40) инвариантна по отношению к левым сдвигам на группе $S L(2, \mathbb{C})$, следовательно, обобщенная функция $(2.45)$ удовлетворяет соотношению ковариантности (2.18).

Докажем абсолютную сходимость интеграла (2.45). Коэффициенты многочлена (2.8) вещественны. Из соотношений $(2.9),(2.10)$ следует равенство

$$
\sum_{k=-l}^{l}\left|t_{m k}^{l}(A)\right|^{2}=t_{m m}^{l}\left(A A^{*}\right)
$$


Из неравенства Коши и формулы (2.46) следует, что

$$
\begin{aligned}
\mid \sum_{k_{1}=-l_{1}}^{l_{1}} \ldots & \sum_{k_{n+1}=-l_{n+1}}^{l_{n+1}} \sum_{\dot{k}_{1}=-i_{1}}^{i_{1}} \ldots \sum_{\dot{k}_{n+1}=-i_{n+1}}^{i_{n+1}}\left(\prod_{i=1}^{n+1} t_{m_{i} k_{i}}^{l_{i}}(g(t, z) u) \overline{t_{\dot{m}_{i} \dot{k}_{i}}^{i_{i}}(g(t, z) u)}\right) \times \\
& \times f_{k_{1}, \ldots, k_{n+1} ; \dot{k}_{1}, \ldots, \dot{k}_{n+1}}^{l_{1}, \ldots, l_{n+1} ; i_{1}, \ldots, i_{n+1}}\left(\mu p_{1}, \ldots, \mu p_{m}\right) \mid \leqslant \\
\leqslant & \sum_{k_{1}=-l_{1}}^{l_{1}} \ldots \sum_{k_{n+1}=-l_{n+1}}^{l_{k_{1}}=-i_{1}} \sum_{\dot{k}_{n+1}=-i_{n+1}}^{i_{1}} \mu^{-2 \sum_{i=1}^{n+1}\left(l_{i}+i_{i}\right)} \times \\
& \times\left.\left|f_{k_{1}, \ldots, k_{n+1} ; \dot{k}_{1}, \ldots, \dot{k}_{n+1}}^{l_{1}, \ldots, l_{n+1} ; i_{1}, \ldots, i_{n+1}}\left(\mu p_{1}, \ldots, \mu p_{m}\right)\right|^{2}\right|^{1 / 2} \times \\
& \times\left(\prod_{i=1}^{n+1} t_{m_{i} m_{i}}^{l_{i}}\left(\mu g(t, z) g(t, z)^{*}\right) t_{\dot{m}_{i} \dot{m}_{i}}^{i_{i_{i}}}\left(\mu g(t, z) g(t, z)^{*}\right)\right)^{1 / 2}
\end{aligned}
$$

Введем переменные

$$
\begin{aligned}
& \tilde{x}_{j}=\mu g(t, z) \tilde{p}_{j} g(t, z)^{*}, \quad j=1, \ldots, m, \\
& \tilde{p}_{1}=\sigma^{0}, \quad \tilde{p}_{m}=\sigma^{0}-\sum_{j=1}^{m-1} \tilde{p}_{j}, \quad m>1 .
\end{aligned}
$$

Суммируя равенства для $\tilde{x}_{j}$ в $(2.48)$, мы получаем разложение $(2.26)$ для матрицы $\tilde{x}_{1}+\cdots+\tilde{x}_{m}$. Функция $f_{m_{1}, \ldots, m_{n+1} ; \dot{m}_{1}, \ldots, \dot{m}_{n+1}}^{l_{1}, \ldots, l_{n+1} ; i_{1}, \ldots, i_{n+1}}\left(p_{1}, \ldots, p_{m}\right)$ полиномиально ограничена. На множестве

$$
\left\{p_{1}, \ldots, p_{m} \in \bar{V}_{+}: \sum_{j=1}^{m} \tilde{p}_{j}=\sigma^{0}\right\}
$$

справедлива следующая оценка:

$$
\left|f_{m_{1}, \ldots, m_{n+1} ; \dot{m}_{1}, \ldots, \dot{m}_{n+1}}^{l_{1}, \ldots, l_{n+1} ; i_{1}, \ldots, i_{n+1}}\left(\mu p_{1}, \ldots, \mu p_{m}\right)\right| \leqslant C\left(1+\sum_{\nu=0}^{3} \sum_{i=1}^{m}\left(\mu p_{i}^{\nu}\right)^{2}\right)^{N} \leqslant C\left(1+4 m \mu^{2}\right)^{N},
$$

где $C$ не зависит от $p_{1}, \ldots, p_{m}$. Функция $f_{m_{1}, \ldots, m_{n+1} ; \dot{m}_{1}, \ldots, \dot{m}_{n+1}}^{l_{1}, \ldots, l_{n+1} ; \dot{l}_{1}, \ldots, i_{n+1}}\left(p_{1}, \ldots, p_{m}\right)$ дифференцируема $l_{1}+\cdots+l_{n+1}+i_{1}+\cdots+i_{n+1}+1$ раз, и ее носитель лежит в произведении $\bar{V}_{+}^{\times m}$ конусов. Следовательно, функция $f_{m_{1}, \ldots, m_{n+1} ; \dot{m}_{1}, \ldots, \dot{m}_{n+1}}^{l_{1}, \ldots, l_{n+1} ; i_{1}, \ldots, i_{n+1}}\left(\mu p_{1}, \ldots, \mu p_{m}\right)$ дифференцируема $l_{1}+\cdots+l_{n+1}+i_{1}+\cdots+i_{n+1}+1$ раз по переменной $\mu$, и ее первые $l_{1}+\cdots+l_{n+1}+i_{1}+\cdots+i_{n+1}$ производных равны нулю в точке $\mu=0$. Тогда из неравенств (2.47), (2.49) следует абсолютная сходимость интеграла (2.45). Интеграл (2.45) определяет обобщенную функцию из $S^{\prime}\left(\mathbb{R}^{4 m}\right)$.

Докажем равенство (2.45). Если носитель обобщенной функции

$$
F_{m_{1}, \ldots, m_{n+1} ; \dot{m}_{1}, \ldots, \dot{m}_{n+1}}^{l_{1}, \ldots, l_{n+1} ; i_{1}, \ldots, i_{n+1}}\left(x_{1}, \ldots, x_{m}\right) \in S^{\prime}\left(\mathbb{R}^{4 m}\right)
$$


лежит в произведении $\bar{V}_{+}^{\times m}$ конусов, то справедливы соотношения, аналогичные равенствам (2.19), (2.24):

$$
\begin{aligned}
& F_{m_{1}, \ldots, m_{n+1} ; \dot{m}_{1}, \ldots, \dot{m}_{n+1}}^{l_{1}, \ldots, l_{n+1} ; i_{1}, \ldots, i_{n+1}}\left(x_{1}, \ldots, x_{m}\right)= \\
& =\left(\prod_{i=1}^{m}\left(\partial_{x_{i}}, \partial_{x_{i}}\right)^{q}\right) f_{m_{1}, \ldots, m_{n+1} ; \dot{m}_{1}, \ldots, \dot{m}_{n+1}}^{l_{1}, \ldots, l_{n+1} ; i_{1}, \ldots, i_{n+1}}\left(x_{1}, \ldots, x_{m}\right), \\
& f_{m_{1}, \ldots, m_{n+1} ; \dot{m}_{1}, \ldots, \dot{m}_{n+1}}^{l_{1}, \ldots, l_{n+1} ; i_{1}, \ldots, i_{n+1}}\left(x_{1}, \ldots, x_{m}\right)=\frac{1}{\left(2 \pi 4^{q-1}(q-2) !(q-1) !\right)^{m}} \times \\
& \quad \times \int d^{4 m} y F_{m_{1}, \ldots, m_{n+1} ; \dot{m}_{1}, \ldots, \dot{m}_{n+1}}^{l_{1}, \ldots, l_{n+1} ; i_{1}, \ldots, i_{n+1}}\left(y_{1}, \ldots, y_{m}\right) \times \\
& \quad \times \prod_{i=1}^{m}\left(x_{i}-y_{i}, x_{i}-y_{i}\right)^{q-2} \theta\left(x_{i}^{0}-y_{i}^{0}\right) \theta\left(\left(x_{i}-y_{i}, x_{i}-y_{i}\right)\right) .
\end{aligned}
$$

В этих формулах можно выбрать такое натуральное число $q$, чтобы функция (2.51) была дифференцируема $l_{1}+\cdots+l_{n+1}+i_{1}+\cdots+i_{n+1}+1$ раз. Носитель этой функции лежит в произведении $\bar{V}_{+}^{\times m}$ конусов, и она полиномиально ограничена. Если обобщенная функция (2.50) удовлетворяет соотношению ковариантности (2.18), то функция (2.51) также удовлетворяет соотношению ковариантности (2.18). Используя доказательство предложения, получаем

$$
\begin{gathered}
f_{m_{1}, \ldots, m_{n+1} ; \dot{m}_{1}, \ldots, \dot{m}_{n+1}}^{l_{1}, \ldots, l_{n+1} ; i_{1}, \ldots, i_{n+1}}\left(\mu g(t, z) \tilde{p}_{1} g(t, z)^{*}, \ldots, \mu g(t, z) \tilde{p}_{m} g(t, z)^{*}\right)= \\
=\sum_{k_{1}=-l_{1}}^{l_{1}} \ldots \sum_{k_{n+1}=-l_{n+1}}^{l_{n+1}} \sum_{\dot{k}_{1}=-i_{1}}^{i_{1}} \ldots \\
\quad \ldots \sum_{\dot{k}_{n+1}=-i_{n+1}}^{i_{n+1}}\left(\prod_{i=1}^{n+1} t_{m_{i} k_{i}}^{l_{i}}(g(t, z)) \overline{t_{\dot{m}_{i} \dot{k}_{i}}^{i_{i}}(g(t, z))}\right) \times \\
\times f_{k_{1}, \ldots, k_{n+1} ; \dot{k}_{1}, \ldots, \dot{k}_{n+1}}^{l_{1}, \ldots, l_{n+1} ; \dot{l}_{1}, \ldots, i_{n+1}}\left(\mu \tilde{p}_{1}, \ldots, \mu \tilde{p}_{m}\right), \\
\tilde{p}_{1}=\sigma^{0}, \quad \tilde{p}_{m}=\sigma^{0}-\sum_{j=1}^{m-1} \tilde{p}_{j}, \quad m>1 .
\end{gathered}
$$

В силу определения (2.51) функция $f_{k_{1}, \ldots, k_{n+1} ; \dot{k}_{1}, \ldots, \dot{k}_{n+1}}^{l_{1}, \ldots, l_{n+1} ; i_{1}, \ldots, i_{n+1}}\left(\mu p_{1}, \ldots, \mu p_{m}\right)$ дифференцируема $l_{1}+\cdots+l_{n+1}+i_{1}+\cdots+i_{n+1}+1$ раз по переменной $\mu$, и ее первые $l_{1}+\cdots+l_{n+1}+$ $\dot{i}_{1}+\cdots+i_{n+1}$ производных равны нулю в точке $\mu=0$. Справедлива оценка (2.49). Используя соотношения $(2.44),(2.52)$ и переменные $(2.48)$, получаем

$$
\begin{gathered}
\int d^{4 m} x f_{m_{1}, \ldots, m_{n+1} ; \dot{m}_{1}, \ldots, \dot{m}_{n+1}}^{l_{1}, \ldots, l_{n+1} ; i_{1}, \ldots, i_{n+1}}\left(x_{1}, \ldots, x_{n+1}\right) \phi\left(\tilde{x}_{1}, \ldots, \tilde{x}_{m}\right)= \\
=\sum_{k_{1}=-l_{1}}^{l_{1}} \ldots \sum_{k_{n+1}=-l_{n+1}}^{l_{n+1}} \sum_{k_{1}=-i_{1}}^{i_{1}} \ldots \sum_{k_{n+1}=-i_{n+1}}^{i_{n+1}} \int_{0}^{\infty} \mu^{4 m-1} d \mu \times
\end{gathered}
$$




$$
\begin{aligned}
& \times \int_{0}^{\infty} t^{-3} d t \int d \operatorname{Re} z \int d \operatorname{Im} z \int d^{4 m} p \delta\left(\sum_{j=1}^{m} \tilde{p}_{j}-\sigma^{0}\right) \times \\
& \times\left(\prod_{i=1}^{n+1} t_{m_{i} k_{i}}^{l_{i}}(g(t, z)) \overline{t_{\dot{m}_{i} \dot{k}_{i}}^{i_{i}}(g(t, z))}\right) 2 f_{k_{1}, \ldots, k_{n+1} ; \dot{k}_{1}, \ldots, \dot{k}_{n+1}}^{l_{1}, \ldots, l_{n+1} ; \dot{l}_{1}, \ldots, \dot{i}_{n+1}}\left(\mu p_{1}, \ldots, \mu p_{m}\right) \times \\
& \times \phi\left(\mu g(t, z) \tilde{p}_{1} g(t, z)^{*}, \ldots, \mu g(t, z) \tilde{p}_{m} g(t, z)^{*}\right) .
\end{aligned}
$$

С помощью соотношения ковариантности (2.18) для функции (2.51) и матрицы $u \in$ $S U(2)$ имеем

$$
\begin{gathered}
f_{m_{1}, \ldots, m_{n+1} ; \dot{m}_{1}, \ldots, \dot{m}_{n+1}}^{l_{1}, \ldots, l_{n+1} ; i_{1}, \ldots, i_{n+1}}\left(\mu \tilde{p}_{1}, \ldots, \mu \tilde{p}_{m}\right)= \\
=\sum_{k_{1}=-l_{1}}^{l_{1}} \ldots \sum_{\substack{k_{n+1}=-l_{n+1} \\
k_{k_{1}}=-i_{1}}}^{l_{n+1}} \ldots \sum_{\dot{k}_{n+1}=-i_{n+1}}^{i_{1}}\left(\prod_{i=1}^{i_{n+1}} t_{m_{i} k_{i}}^{l_{i}}(u) \overline{t_{\dot{m}_{i} \dot{k}_{i}}^{\dot{l}_{i}}(u)}\right) \times \\
\quad \times f_{k_{1}, \ldots, k_{n+1} ; \dot{k}_{1}, \ldots, \dot{k}_{n+1}}^{l_{1}, \ldots, l_{n+1} ; i_{1}, \ldots, i_{n+1}}\left(\mu u^{-1} \tilde{p}_{1} u, \ldots, \mu u^{-1} \tilde{p}_{m} u\right), \\
\tilde{p}_{1}=\sigma^{0}, \quad \tilde{p}_{m}=\sigma^{0}-\sum_{j=1}^{m-1} \tilde{p}_{j}, \quad m>1 .
\end{gathered}
$$

Проинтегрируем равенство (2.54) с нормированной мерой Хаара на группе $S U(2)$ и подставим полученное равенство в (2.53). Сделаем замену переменных интегрирования

$$
\begin{gathered}
\tilde{p}_{j} \rightarrow u \tilde{p}_{j} u^{*}, \quad j=1, \ldots, m-1, \quad m>1, \\
u \tilde{p}_{m} u^{*}=\tilde{p}_{m}=\sigma_{0}, \quad m=1 .
\end{gathered}
$$

Равенство, полученное после интегрирования, и соотношение (2.50) дают (2.45).

С помощью формул (2.11), (2.13) можно вычислить интеграл по группе $S U(2)$ в правой части равенства $(2.45)$ для $m=n=1$. Полученное равенство может быть переписано в виде (2.25).

Можно получить интегральное представление для преобразования Фурье-Лапласа обобщенной функции (2.45). Для двумерного пространства-времени подобное интегральное представление было получено Жариновым [11]. Аналитические свойства преобразования Фурье-Лапласа обобщенной функции (2.45) изучались раньше с помощью теоремы Баргмана-Холла-Вайтмана [2]-[4]. Интегральное представление для преобразования Фурье-Лапласа обобщенной функции (2.45) упрощает эту задачу.

Вакуумное ожидание произведения двух квантовых полей является преобразованием Фурье обобщенной функции (2.25). Представим обобщенную функцию (2.25) в виде, удобном для вычисления преобразования Фурье. Используя матрицы (2.3) как коэффициенты, определим $(2 \times 2)$-матрицу

$$
\tilde{\partial}_{x}=\sum_{\mu=0}^{3} \eta^{\mu \mu} \sigma^{\mu} \frac{\partial}{\partial x^{\mu}},
$$


где $\eta^{00}=-\eta^{11}=-\eta^{22}=-\eta^{33}=1$. Подставляя эту матрицу в многочлен $(2.8)$, получим дифференциальный оператор $t_{m n}^{l}\left(\tilde{\partial}_{x}\right)$.

ЛЕмма 2. Пусть функиия $f(s)$ дифференцируема $2 l$ раз. Тогда

$$
t_{m n}^{l}\left(\tilde{\partial}_{x}\right) f((x, x))=\left.t_{m n}^{l}(\tilde{x})\left(2 \frac{d}{d s}\right)^{2 l} f(s)\right|_{s=(x, x)} .
$$

ДоКАЗАТЕЛЬСтво. Из определений (2.3), (2.8), (2.55) следует, что

$$
\begin{aligned}
& t_{m n}^{l}\left(\tilde{\partial}_{x}\right) f((x, x))=\sqrt{(l-m) !(l+m) !(l-n) !(l+n) !} \times \\
& \quad \times \sum_{j=-\infty}^{\infty} \frac{1}{\Gamma(j+1) \Gamma(l-m-j+1) \Gamma(m-n+j+1) \Gamma(l+n-j+1)} \times \\
& \quad \times\left(\frac{\partial}{\partial x^{0}}-\frac{\partial}{\partial x^{3}}\right)^{l-m-j}\left(-\frac{\partial}{\partial x^{1}}+i \frac{\partial}{\partial x^{2}}\right)^{j} \times \\
& \quad \times\left(\left.\left(x^{1}+i x^{2}\right)^{m-n+j}\left(x^{0}-x^{3}\right)^{l+n-j}\left(2 \frac{d}{d s}\right)^{l+m} f(s)\right|_{s=(x, x)}\right) .
\end{aligned}
$$

С помощью правила Лейбница получаем

$$
\begin{aligned}
\left(\frac{\partial}{\partial x^{0}}-\right. & \left.\frac{\partial}{\partial x^{3}}\right)^{l-m-j}\left(-\frac{\partial}{\partial x^{1}}+i \frac{\partial}{\partial x^{2}}\right)^{j} \times \\
& \times\left(\left.\left(x^{1}+i x^{2}\right)^{m-n+j}\left(x^{0}-x^{3}\right)^{l+n-j}\left(2 \frac{d}{d s}\right)^{l+m} f(s)\right|_{s=(x, x)}\right)= \\
= & \sum_{p=-\infty}^{\infty} \sum_{q=-\infty}^{\infty} \frac{\Gamma(l-m-j+1) \Gamma(j+1)}{\Gamma(p+1) \Gamma(l-m-j-p+1) \Gamma(q+1) \Gamma(j-q+1)} \times \\
& \times\left(\left(\frac{\partial}{\partial x^{0}}-\frac{\partial}{\partial x^{3}}\right)^{p}\left(x^{0}-x^{3}\right)^{l+n-j}\right)\left(\left(-\frac{\partial}{\partial x^{1}}+i \frac{\partial}{\partial x^{2}}\right)^{q}\left(x^{1}+i x^{2}\right)^{m-n+j}\right) \times \\
& \times\left.\left(\frac{\partial}{\partial x^{0}}-\frac{\partial}{\partial x^{3}}\right)^{l-m-j-p}\left(-\frac{\partial}{\partial x^{1}}+i \frac{\partial}{\partial x^{2}}\right)^{j-q}\left(2 \frac{d}{d s}\right)^{l+m} f(s)\right|_{s=(x, x)} \cdot(2.58)
\end{aligned}
$$

Вычисляя производные в правой части равенства (2.58), можно переписать (2.57) в виде

$$
\begin{aligned}
& t_{m n}^{l}\left(\tilde{\partial}_{x}\right) f((x, x))=\sqrt{(l-m) !(l+m) !(l-n) !(l+n) !} \times \\
& \quad \times \sum_{j=-\infty}^{\infty} \sum_{p=-\infty}^{\infty} \sum_{q=-\infty}^{\infty}(-1)^{q} 2^{p+q} \frac{1}{\Gamma(p+1) \Gamma(q+1)} \times \\
& \quad \times \frac{1}{\Gamma(j-q+1) \Gamma(l-m-j-p+1) \Gamma(m-n+j-q+1) \Gamma(l+n-j-p+1)} \times \\
& \quad \times\left(x^{0}+x^{3}\right)^{l-m-j-p}\left(x^{0}-x^{3}\right)^{l+n-j-p}\left(x^{1}+i x^{2}\right)^{m-n+j-q} \times \\
& \quad \times\left.\left(x^{1}-i x^{2}\right)^{j-q}\left(2 \frac{d}{d s}\right)^{2 l-p-q} f(s)\right|_{s=(x, x)} .
\end{aligned}
$$


Делая замену $j \rightarrow j+q, p \rightarrow p-q$ переменных суммирования, получаем

$$
\begin{aligned}
& t_{m n}^{l}\left(\tilde{\partial}_{x}\right) f((x, x))=\sqrt{(l-m) !(l+m) !(l-n) !(l+n) !} \times \\
& \quad \times \sum_{j=-\infty}^{\infty} \sum_{p=-\infty}^{\infty} \sum_{q=-\infty}^{\infty}(-1)^{q} 2^{p} \frac{1}{\Gamma(p-q+1) \Gamma(q+1)} \times \\
& \quad \times \frac{1}{\Gamma(j+1) \Gamma(l-m-j-p+1) \Gamma(m-n+j+1) \Gamma(l+n-j-p+1)} \times \\
& \quad \times\left(x^{0}+x^{3}\right)^{l-m-j-p}\left(x^{0}-x^{3}\right)^{l+n-j-p}\left(x^{1}+i x^{2}\right)^{m-n+j} \times \\
& \quad \times\left.\left(x^{1}-i x^{2}\right)^{j}\left(2 \frac{d}{d s}\right)^{2 l-p} f(s)\right|_{s=(x, x)} .
\end{aligned}
$$

Справедливо тождество

$$
\sum_{q=0}^{p}(-1)^{q} \frac{\Gamma(p+1)}{\Gamma(p-q+1) \Gamma(q+1)}=(1-1)^{p}
$$

При $p \geqslant 1$ обе части этого равенства равны нулю. При $p=0$ левая часть равенства равна 1. С помощью формулы (2.61) мы можем переписать равенство (2.60) в виде (2.56).

Определим

$$
f^{(-2 l)}(s)=\theta(s) \int_{0}^{s} d t \frac{(s-t)^{2 l-1}}{(2 l-1) !} f\left(t^{1 / 2}\right), \quad 2 l \geqslant 1, \quad f^{(0)}(s)=\theta(s) f\left(s^{1 / 2}\right) .
$$

Отсюда с помощью равенства (2.56) получаем

$$
t_{m n}^{l}(\tilde{x}) \theta((x, x)) f\left((x, x)^{1 / 2}\right)=2^{-2 l} t_{m n}^{l}\left(\tilde{\partial}_{x}\right) f^{(-2 l)}((x, x))
$$

Если функция $f(s)$ полиномиально ограничена, то функция (2.62) также полиномиально ограничена. Если функция $f(s)$ непрерывна и ее носитель лежит на положительной полуоси, то функция (2.62) также непрерывна и ее носитель лежит на положительной полуоси. Функция (2.62) дифференцируема $2 l$ раз, и ее первые $2 l$ производных равны нулю в точке $s=0$. Из (2.63) вытекает

$$
\begin{aligned}
& \theta\left(x^{0}\right) \theta((x, x)) t_{m_{3} \dot{m}_{3}}^{l_{3}}(\tilde{x}) f^{l_{1}, l_{2}, l_{3} ; i_{1}, i_{2}}\left((x, x)^{1 / 2}\right)= \\
& \quad=t_{m_{3} \dot{m}_{3}}^{l_{3}}\left(\tilde{\partial}_{x}\right)\left(\theta\left(x^{0}\right) 2^{-2 l_{3}}\left(f^{l_{1}, l_{2}, l_{3} ; l_{1}, l_{2}}\right)^{\left(-2 l_{3}\right)}((x, x))\right) .
\end{aligned}
$$

Подставляя это равенство в соотношение (2.25), получаем произвольную обобщенную функцию

$$
F_{m_{1}, m_{2} ; \dot{m}_{1}, \dot{m}_{2}}^{l_{1}, l_{2} ; i_{1}, i_{2}}(x) \in S^{\prime}\left(\mathbb{R}^{4}\right)
$$


с носителем в замкнутом верхнем световом конусе, удовлетворяющую соотношению ковариантности (2.18) для $m=n=1$, в следующем виде:

$$
\begin{aligned}
& \int d^{4} x F_{m_{1}, m_{2} ; \dot{m}_{1}, \dot{m}_{2}}^{l_{1}, l_{2} ; i_{1}, i_{2}}(x) \phi(x)= \\
&= \sum_{l_{3} \in \mathbb{Z}_{+} / 2} \sum_{m_{3}, \dot{m}_{3}=-l_{3}}^{l_{3}} C\left(l_{1}, l_{2}, l_{3} ; m_{1}, m_{2}, m_{3}\right) C\left(\dot{l}_{1}, \dot{l}_{2}, l_{3} ; \dot{m}_{1}, \dot{m}_{2}, \dot{m}_{3}\right) \times \\
& \quad \times \int d^{4} x \theta\left(x^{0}\right) 2^{-2 l_{3}}\left(f^{l_{1}, l_{2}, l_{3} ; i_{1}, i_{2}}\right)^{\left(-2 l_{3}\right)}((x, x))\left(\partial_{x}, \partial_{x}\right)^{q} t_{m_{3} \dot{m}_{3}}^{l_{3}}\left(-\tilde{\partial}_{x}\right) \phi(x),
\end{aligned}
$$

где функция $\phi(x) \in S\left(\mathbb{R}^{4}\right), q$ - натуральное число, коэффициент Клебша-Гордана $C\left(l_{1}, l_{2}, l_{3} ; m_{1}, m_{2}, m_{3}\right)$ задан соотношением $(2.12),(2 \times 2)$-матрица $\tilde{\partial}_{x}$ - соотношением (2.55), многочлен $t_{m n}^{l}(A)$ - соотношением (2.8), непрерывная функция $\left(f^{l_{1}, l_{2}, l_{3} ; \dot{l}_{1}, i_{2}}\right)^{\left(-2 l_{3}\right)}(s)$ с носителем на положительной полуоси полиномиально ограничена.

Соотношение (2.64) является разложением по конечномерным неприводимым представлениям группы $S L(2, \mathbb{C})$. Рассмотрим обобщенные функции умеренного роста следующего вида:

$$
\begin{aligned}
& \int d^{4 m} x F_{m_{1}, \ldots, m_{n+1} ; \dot{m}_{1}, \ldots, \dot{m}_{n+1}}^{l_{1}, \ldots, l_{n+1} ; i_{1}, \ldots, i_{n+1}}\left(x_{1}, \ldots, x_{m}\right) \phi\left(x_{1}, \ldots, x_{m}\right)= \\
& =\sum_{l_{n+2}, \ldots, l_{n+m+1} \in \mathbb{Z}_{+} / 2} \sum_{\substack{m_{s}, \dot{m}_{s}=-l_{s},-l_{s}+1, \ldots, l_{s}-1, l_{s} \\
s=n+2, \ldots, n+m+1}} \\
& \int d^{4 m} x f_{m_{1}, \ldots, m_{n+m+1} ; \dot{m}_{1}, \ldots, \dot{m}_{n+m+1}}^{l_{1}, \ldots, l_{n+m+1} ; i_{1}, \ldots, i_{n+1}}\left(\left(x_{1}, x_{1}\right), \ldots,\left(x_{m}, x_{m}\right)\right) \times \\
& \times\left(\prod_{j=1}^{m} \theta\left(x_{j}^{0}\right)\left(\partial_{x_{j}}, \partial_{x_{j}}\right)^{q} t_{m_{n+1+j} \dot{m}_{n+1+j}}^{l_{n+1+j}}\left(-\tilde{\partial}_{x_{j}}\right)\right) \phi\left(x_{1}, \ldots, x_{m}\right),
\end{aligned}
$$

где $\phi\left(x_{1}, \ldots, x_{m}\right) \in S\left(\mathbb{R}^{4}\right), q$ - натуральное число, $(2 \times 2)$-матрица $\tilde{\partial}_{x}$ задана соотношением (2.55), многочлен $t_{m n}^{l}(A)$ - соотношением $(2.8)$, непрерывная функция

$$
f_{m_{1}, \ldots, m_{n+m+1} ; \dot{m}_{1}, \ldots, \dot{m}_{n+m+1}}^{l_{1}, \ldots, l_{n+m+1} ; i_{1}, \ldots, i_{n+1}}\left(s_{1}, \ldots, s_{m}\right)
$$

с носителем в произведении положительных полуосей полиномиально ограничена; суммы по переменным $l_{n+2}, \ldots, l_{n+m+1}$ конечны. Обобщенная функция (2.65) принадлежит пространству $S^{\prime}\left(\mathbb{R}^{4 m}\right)$. Сумма по переменной $l_{3}$ в соотношении $(2.64)$ конечна в силу условия треугольника для коэффициентов Клебша-Гордана.

Определим для любых чисел $m, n \in \mathbb{Z}_{+}$обобщенный коэффициент КлебшаГордана

$$
\begin{aligned}
C\left(l_{1}, \ldots,\right. & \left.l_{m+2} ; l_{m+3}, \ldots, l_{m+n+4} ; j_{1}, \ldots, j_{m+n+1} ; m_{1}, \ldots, m_{m+2} ; m_{m+3}, \ldots, m_{m+n+4}\right)= \\
= & \sum_{\substack{k_{s}=-j_{s},-j_{s}+1, \ldots, j_{s}-1, j_{s} \\
s=1, \ldots, m+n+1}} C\left(l_{1}, l_{2}, j_{1} ; m_{1}, m_{2}, k_{1}\right) \times \\
& \times\left(\prod_{s=1}^{m} C\left(j_{s}, l_{s+2}, j_{s+1} ; k_{s}, m_{s+2}, k_{s+1}\right)\right) \times
\end{aligned}
$$




$$
\begin{aligned}
& \times\left(\prod_{s=m+1}^{m+n} C\left(j_{s+1}, l_{s+2}, j_{s} ; k_{s+1}, m_{s+2}, k_{s}\right)\right) \times \\
& \times C\left(l_{m+n+4}, l_{m+n+3}, j_{m+n+1} ; m_{m+n+4}, m_{m+n+3}, k_{m+n+1}\right),
\end{aligned}
$$

где числа $l_{1}, \ldots, l_{m+n+4}, j_{1}, \ldots, j_{m+n+1} \in \mathbb{Z}_{+} / 2$ и $m_{i}=-l_{i},-l_{i}+1, \ldots, l_{i}-1, l_{i}$, $i=1, \ldots, m+n+4$. Из определения (2.66) и формул (2.10), (2.17) следуют такие соотношения для любой матрицы $A \in S L(2, \mathbb{C})$ :

$$
\begin{aligned}
& \sum_{\substack{n_{s}=-l_{s},-l_{s}+1, \ldots, l_{s}-1, l_{s}, s=1, \ldots, m+2 \mid}}\left(\prod_{i=1}^{m+2} t_{m_{i} n_{i}}^{l_{i}}(A)\right) \times \\
& \times C\left(l_{1}, \ldots, l_{m+2} ; l_{m+3}, \ldots, l_{m+n+4} ; j_{1}, \ldots, j_{m+n+1} ; n_{1}, \ldots, n_{m+2} ; m_{m+3}, \ldots, m_{m+n+4}\right)= \\
& =\sum_{\substack{n_{s}=-l_{s},-l_{s}+1, \ldots, l_{s}-1, l_{s} \\
s=m+3, \ldots, m+n+4}}\left(\prod_{i=m+3}^{m+n+4} t_{n_{i} m_{i}}^{l_{i}}(A)\right) \times
\end{aligned}
$$$$
\times C\left(l_{1}, \ldots, l_{m+2} ; l_{m+3}, \ldots, l_{m+n+4} ; j_{1}, \ldots, j_{m+n+1} ; m_{1}, \ldots, m_{m+2} ; n_{m+3}, \ldots, n_{m+n+4}\right) \text {, }
$$

$$
\sum_{\substack{n_{s}=-l_{s},-l_{s}+1, \ldots, l_{s}-1, l_{s}, s=1, \ldots, m+2}}\left(\prod_{i=1}^{m+2} t_{n_{i} m_{i}}^{l_{i}}(A)\right) \times
$$

$\times C\left(l_{1}, \ldots, l_{m+2} ; l_{m+3}, \ldots, l_{m+n+4} ; j_{1}, \ldots, j_{m+n+1} ; n_{1}, \ldots, n_{m+2} ; m_{m+3}, \ldots, m_{m+n+4}\right)=$

$$
=\sum_{\substack{n_{s}=-l_{s},-l_{s}+1, \ldots, l_{s}-1, l_{s} \\ s=m+3, \ldots, m+n+4}}\left(\prod_{i=m+3}^{m+n+4} t_{m_{i} n_{i}}^{l_{i}}(A)\right) \times
$$

$\times C\left(l_{1}, \ldots, l_{m+2} ; l_{m+3}, \ldots, l_{m+n+4} ; j_{1}, \ldots, j_{m+n+1} ; m_{1}, \ldots, m_{m+2} ; n_{m+3}, \ldots, n_{m+n+4}\right)$.

ТЕорема 2. Если обобщенные функиии (2.65) для $m=n=1$ удовлетворяют соотношению ковариантности (2.18), то

$f_{m_{1}, m_{2}, m_{3} ; \dot{m}_{1}, \dot{m}_{2}, \dot{m}_{3}}^{l_{1}, l_{2}, l_{3} ; i_{1}, i_{2}}\left(s_{1}\right)=f^{l_{1}, l_{2}, l_{3} ; \dot{l}_{1}, \dot{l}_{2}}\left(s_{1}\right) C\left(l_{1}, l_{2}, l_{3} ; m_{1}, m_{2}, m_{3}\right) C\left(\dot{l}_{1}, \dot{l}_{2}, l_{3} ; \dot{m}_{1}, \dot{m}_{2}, \dot{m}_{3}\right)$,

где непрерывная функиия $f^{l_{1}, l_{2}, l_{3} ; i_{1}, l_{2}}\left(s_{1}\right)$ с носителем на положителъной полуоси полиномиально ограничена, коэффициент $C\left(l_{1}, l_{2}, l_{2}, l_{3} ; m_{1}, m_{2}, m_{3}\right)$ задан соотношением (2.12).

Если обобщенные функиии (2.65) для $m \geqslant 2, n \geqslant 1$ удовлетворяют соотношению ковариантности (2.18), то

$$
\begin{aligned}
& f_{m_{1}, \ldots, m_{n+m+1} ; m_{1}, \ldots, m_{n+m+1}}^{l_{1}, \ldots, l_{n+m+1} ; i_{1}, \ldots, i_{n+1}}\left(s_{1}, \ldots, s_{m}\right)= \\
& \quad=\sum_{j_{1}, \ldots, j_{n+m-2} ; j_{1}^{\prime}, \ldots, j_{n+m-2}^{\prime} \in \mathbb{Z}_{+} / 2}^{f_{j_{1}, \ldots, j_{n+m-2} ; j_{1}^{\prime}, \ldots, j_{n+m-2}^{\prime}}^{l_{1}, \ldots, l_{n+m+1} ; i_{1}, \ldots, i_{n+1}}\left(s_{1}, \ldots, s_{m}\right) \times} \\
& \times C\left(l_{1}, \ldots, l_{n+1} ; l_{n+2}, \ldots, l_{m+n+1} ; j_{1}, \ldots, j_{m+n-2} ; m_{1}, \ldots, m_{n+1} ; m_{n+2}, \ldots, m_{m+n+1}\right) \times
\end{aligned}
$$


$\times C\left(\dot{l}_{1}, \ldots, \dot{l}_{n+1} ; l_{n+2}, \ldots, l_{m+n+1} ; j_{1}^{\prime}, \ldots, j_{m+n-2}^{\prime} ; \dot{m}_{1}, \ldots, \dot{m}_{n+1} ; \dot{m}_{n+2}, \ldots, \dot{m}_{m+n+1}\right)$,

где непрерывная функиия

$$
f_{j_{1}, \ldots, j_{n+m-2} ; j_{1}^{\prime}, \ldots, j_{n+m-2}^{\prime}}^{l_{1}, \ldots, l_{n+m+1} ; i_{1}, \ldots, i_{n+1}}\left(s_{1}, \ldots, s_{m}\right)
$$

с носителем в произведении положительных полуосей полиномиально ограничена, обобщенный коэфбициент Клебша-Гордана

$$
C\left(l_{1}, \ldots, l_{n+1} ; l_{n+2}, \ldots, l_{m+n+1} ; j_{1}, \ldots, j_{m+n-2} ; m_{1}, \ldots, m_{n+1} ; m_{n+2}, \ldots, m_{m+n+1}\right)
$$

задан соотношением (2.66).

ДокАЗАтеЛьство. Соотношение ковариантности (2.18) может быть переписано в виде

$$
\begin{aligned}
& F_{m_{1}, \ldots, m_{n+1} ; \dot{m}_{1}, \ldots, \dot{m}_{n+1}}^{l_{1}, \ldots, l_{n+1} ; i_{1}, \ldots, i_{n+1}}\left(\tilde{x}_{1}, \ldots, \tilde{x}_{m}\right)= \\
& =\sum_{k_{1}=-l_{1}}^{l_{1}} \ldots \sum_{\substack{k_{n+1}=-l_{n+1} \\
k_{k_{1}}=-\dot{l}_{1}}}^{l_{n+1}} \sum_{\dot{k}_{n+1}=-i_{n+1}}^{i_{1}}\left(\prod_{i=1}^{l_{n+1}} t_{m_{i} k_{i}}^{l_{i}}\left(A^{-1}\right) t_{\dot{m}_{i} \dot{k}_{i}}^{i_{i}}\left(\bar{A}^{-1}\right)\right) \times \\
& \quad \times F_{k_{1}, \ldots, k_{n+1} ; \dot{k}_{1}, \ldots, \dot{k}_{n+1}}^{l_{1}, \ldots, l_{n+1} ; i_{1}, \ldots, i_{n+1}}\left(A \tilde{x}_{1} A^{*}, \ldots, A \tilde{x}_{m} A^{*}\right) .
\end{aligned}
$$

Тогда из равенства (2.65) и соотношений (2.9), (2.10) следует

$$
\begin{aligned}
& \int d^{4 m} x F_{m_{1}, \ldots, m_{n+1} ; \dot{m}_{1}, \ldots, \dot{m}_{n+1}}^{l_{1}, \ldots, l_{n+1} ; i_{1}, \ldots, i_{n+1}}\left(x_{1}, \ldots, x_{m}\right) \phi\left(x_{1}, \ldots, x_{m}\right)= \\
& =\sum_{l_{n+2}, \ldots, l_{n+m+1} \in \mathbb{Z}_{+} / 2} \sum_{\substack{m_{s}, \dot{m}_{s}, k_{s}, \dot{k}_{s}=-l_{s},-l_{s}+1, \ldots, l_{s}-1, l_{s}, s=n+2, \ldots, n+m+1}} \times \\
& \times \sum_{\substack{k_{s}=-l_{s},-l_{s}+1, \ldots, l_{s}-1, l_{s}, \dot{k}_{s}=-i_{s},-i_{s}+1, \ldots, i_{s}-1, i_{s}, s=1, \ldots, n+1}} \times \\
& \times\left(\prod_{i=1}^{n+1} t_{m_{i} k_{i}}^{l_{i}}\left(A^{-1}\right) t_{\dot{m}_{i} \dot{k}_{i}}^{i_{i}}\left(\bar{A}^{-1}\right)\right)\left(\prod_{i=n+2}^{n+m+1} t_{m_{i} k_{i}}^{l_{i}}(A) t_{\dot{m}_{i} \dot{k}_{i}}^{l_{i}}(\bar{A})\right) \times \\
& \times \int d^{4 m} x f_{k_{1}, \ldots, k_{n+m+1} ; \dot{k}_{1}, \ldots, \dot{k}_{n+m+1}}^{l_{1}, \ldots, l_{n+m+1} ; \dot{l}_{1}, \ldots, i_{n+1}}\left(\left(x_{1}, x_{1}\right), \ldots,\left(x_{m}, x_{m}\right)\right) \times \\
& \times\left(\prod_{j=1}^{m} \theta\left(x_{j}^{0}\right)\left(\partial_{x_{j}}, \partial_{x_{j}}\right)^{q} t_{k_{n+1+j} k_{n+1+j}}^{l_{n+1+j}}\left(-\tilde{\partial}_{x_{j}}\right)\right) \phi\left(x_{1}, \ldots, x_{m}\right)
\end{aligned}
$$

для любой матрицы $A \in S L(2, \mathbb{C})$. Суммы в $(2.72)$ по $l_{n+2}, \ldots, l_{n+m+1}$ конечны, следовательно, правая часть равенства (2.72) является многочленом от матричных элементов матриц $A, \bar{A}$. Выберем следующую матрицу из группы $S L(2, \mathbb{C})$ :

$$
A=\exp \left\{\frac{1}{4}\left(\phi_{1}^{\prime}+i \phi_{1}\right) \sigma^{3}\right\} \exp \left\{\frac{1}{4}\left(\theta_{1}^{\prime}+i \theta_{1}\right) \sigma^{1}\right\} \exp \left\{\frac{1}{4}\left(\psi_{1}^{\prime}+i \psi_{1}\right) \sigma^{3}\right\} \times
$$




$$
\times \exp \left\{\frac{1}{4}\left(\phi_{2}^{\prime}-i \phi_{2}\right) \sigma^{3}\right\} \exp \left\{\frac{1}{4}\left(\theta_{2}^{\prime}-i \theta_{2}\right) \sigma^{1}\right\} \exp \left\{\frac{1}{4}\left(\psi_{2}^{\prime}-i \psi_{2}\right) \sigma^{3}\right\} .
$$

По определению (2.3) матричные элементы матриц $\sigma^{1}, \sigma^{3}$ вещественны. Следовательно,

$$
\begin{aligned}
\bar{A}=\exp & \left\{\frac{1}{4}\left(\phi_{1}^{\prime}-i \phi_{1}\right) \sigma^{3}\right\} \exp \left\{\frac{1}{4}\left(\theta_{1}^{\prime}-i \theta_{1}\right) \sigma^{1}\right\} \exp \left\{\frac{1}{4}\left(\psi_{1}^{\prime}-i \psi_{1}\right) \sigma^{3}\right\} \times \\
& \times \exp \left\{\frac{1}{4}\left(\phi_{2}^{\prime}+i \phi_{2}\right) \sigma^{3}\right\} \exp \left\{\frac{1}{4}\left(\theta_{2}^{\prime}+i \theta_{2}\right) \sigma^{1}\right\} \exp \left\{\frac{1}{4}\left(\psi_{2}^{\prime}+i \psi_{2}\right) \sigma^{3}\right\} .
\end{aligned}
$$

Матрицы (2.73), (2.74) аналитически продолжаются на значения параметров $\phi_{k}^{\prime}=$ $i \phi_{k}, \theta_{k}^{\prime}=i \theta_{k}, \psi_{k}^{\prime}=i \psi_{k}, k=1,2$ :

$$
\begin{gathered}
A=u\left(\phi_{1}, \theta_{1}, \psi_{1}\right), \quad \bar{A}=u\left(\phi_{2}, \theta_{2}, \psi_{2}\right), \\
u(\phi, \theta, \psi)=\exp \left\{i \frac{\phi}{2} \sigma^{3}\right\} \exp \left\{i \frac{\theta}{2} \sigma^{1}\right\} \exp \left\{i \frac{\psi}{2} \sigma^{3}\right\} .
\end{gathered}
$$

Согласно [9] (глава III, раздел 6.1) нормированная мера Хаара на группе $S U(2)$ имеет вид

$$
d u(\phi, \theta, \psi)=\frac{1}{16 \pi^{2}} \sin \theta d \theta d \phi d \psi .
$$

Подставим матрицы (2.75) в соотношение (2.72) и проинтегрируем полученное равенство по обеим группам $S U(2)$ с мерами (2.76). Получим

$$
\begin{aligned}
& \int d^{4 m} x F_{m_{1}, \ldots, m_{n+1} ; \dot{m}_{1}, \ldots, \dot{m}_{n+1}}^{l_{1}, \ldots, l_{n+1} ; i_{1}, \ldots, i_{n+1}}\left(x_{1}, \ldots, x_{m}\right) \phi\left(x_{1}, \ldots, x_{m}\right)= \\
&=\sum_{l_{n+2}, \ldots, l_{n+m+1} \in \mathbb{Z}_{+} / 2} \sum_{\substack{m_{s}, \dot{m}_{s}, k_{s}, \dot{k}_{s}=-l_{s},-l_{s}+1, \ldots, l_{s}-1, l_{s}, s=n+2, \ldots, n+m+1}} \sum_{k_{s}=-l_{s},-l_{s}+1, \ldots, l_{s}-1, l_{s}, \dot{k}_{s}=-i_{s},-i_{s}+1, \ldots, i_{s}-1, i_{s},} \times 1, \ldots, n+1 \\
& \times \int_{S U(2)} d u_{1} \int_{S U(2)} d u_{2}\left(\prod_{i=1}^{n+1} t_{m_{i} k_{i}}^{l_{i}}\left(u_{1}^{-1}\right) t_{\dot{m}_{i} \dot{k}_{i}}^{i_{i}}\left(u_{2}^{-1}\right)\right) \times \\
& \times\left(\prod_{i=n+2}^{n+m+1} t_{m_{i} k_{i}}^{l_{i}}\left(u_{1}\right) t_{\dot{m}_{i} \dot{k}_{i}}^{i_{i}}\left(u_{2}\right)\right) \times \\
& \times \int^{4 m} x f_{k_{1}, \ldots, k_{n+m+1} ; \dot{k}_{1}, \ldots, \dot{k}_{n+m+1}}^{l_{1}, \ldots, l_{n+m+1} ; i_{1}, \ldots, i_{n+1}}\left(\left(x_{1}, x_{1}\right), \ldots,\left(x_{m}, x_{m}\right)\right) \times \\
& \times\left(\prod_{j=1}^{m} \theta\left(x_{j}^{0}\right)\left(\partial_{x_{j}}, \partial_{x_{j}}\right)^{q} t_{k_{n+1+j} \dot{k}_{n+1+j}}^{l_{n+1+j}}\left(-\tilde{\partial}_{x_{j}}\right)\right) \phi\left(x_{1}, \ldots, x_{m}\right) .
\end{aligned}
$$

Рассмотрим случай $m=n=1$. В силу определения группы $S U(2)$ имеем $u^{-1}=u^{*}$, поэтому из $(2.10),(2.13),(2.77)$ следует равенство (2.69). В силу $(2.10),(2.17)$ любая 
обобщенная функция (2.65) с функциями (2.69) удовлетворяет соотношению ковариантности (2.18).

Рассмотрим случай $m \geqslant 2, n \geqslant 1$. С помощью формул $(2.10),(2.11),(2.13)$ и (2.77) получаем равенство (2.70). В силу (2.67), (2.68) любая обобщенная функция (2.65) с функциями (2.70) удовлетворяет соотношению ковариантности (2.18).

Если мы подставим функции (2.69) в ряд (2.65) для $m=n=1$, то этот ряд будет конечным в силу условия треугольника для коэффициентов Клебша-Гордана. Если мы подставим функции (2.70) в ряд (2.65) для $m \geqslant 2, n \geqslant 1$, то этот ряд может быть бесконечен. Необходимо выбрать функции $\phi\left(x_{1}, \ldots, x_{m}\right)$, для которых ряд сходится. Если преобразование Фурье

$$
\tilde{\phi}\left(p_{1}, \ldots, p_{m}\right)=\int d^{4 m} x \exp \left\{i \sum_{j=1}^{m}\left(p_{j}, x_{j}\right)\right\} \phi\left(x_{1}, \ldots, x_{m}\right)
$$

принадлежит пространству $D\left(\mathbb{R}^{4 m}\right)$, то функция (2.78) бесконечно дифференцируема и имеет компактный носитель.

В силу соотношения (2.23) кажется естественным рассмотреть обобщенную функцию (2.65) с коэффициентными функциями

$$
\begin{aligned}
& f_{m_{1}, \ldots, m_{n+m+1} ; \dot{m}_{1}, \ldots, \dot{m}_{n+m+1}}^{l_{1}, \ldots, l_{n+m+1} ; i_{1}, \ldots, i_{n+1}}\left(\left(x_{1}, x_{1}\right), \ldots,\left(x_{m}, x_{m}\right)\right)= \\
& \quad=a_{m_{1}, \ldots, m_{n+m+1} ; \dot{m}_{1}, \ldots, \dot{m}_{n+m+1}}^{l_{1}, \ldots, l_{n+m+1} ; \dot{l}_{1}, \ldots, i_{n+1}} \prod_{j=1}^{m}\left(\left(x_{j}, x_{j}\right)^{q-2} \theta\left(\left(x_{j}, x_{j}\right)\right)\right) .
\end{aligned}
$$

Подстановка равенств (2.79) в ряд (2.65) дает

$$
\left.\sum_{\substack{k_{j}^{\mu} \in \mathbb{Z}_{+}, j=1, \ldots, m, \mu=0, \ldots, 3}} a\left(k_{j}^{\mu}\right)\left(\prod_{j=1}^{m} \prod_{\mu=0}^{3}\left(\frac{\partial}{\partial x_{j}^{\mu}}\right)^{k_{j}^{\mu}}\right) \phi\left(x_{1}, \ldots, x_{m}\right)\right|_{x_{j}=0} .
$$

Если ряд (2.80) сходится для любой функции $\phi\left(x_{1}, \ldots, x_{m}\right) \in D\left(\mathbb{R}^{4 m}\right)$, то согласно теореме Бореля (см. [12], раздел 1.5) число отличных от нуля коэффициентов $a\left(k_{j}^{\mu}\right)$ конечно.

Преобразование Фурье обобщенной функции (2.80) является степенным рядом. Поэтому естественно определить ряд (2.80) для функций $\phi\left(x_{1}, \ldots, x_{m}\right) \in S\left(\mathbb{R}^{4 m}\right), \mathrm{y}$ которых преобразования Фурье (2.78) имеют компактные носители.

Аналогичный результат можно получить с помощью теоремы о конечной ковариантности. Пусть функция $f\left(z_{1}, \ldots, z_{m}\right)$ голоморфна в $m$-точечной расширенной трубе

$$
T_{m}^{\prime}=\left\{z \in \mathbb{C}^{4 m}: \tilde{z}_{k}=A\left(\tilde{x}_{k}+i \tilde{y}_{k}\right) B, y_{k} \in V_{+}, k=1, \ldots, m, A, B \in S L(2, \mathbb{C})\right\} .
$$

Пусть ее ограничения на

$$
T_{m}^{+}=\left\{z \in \mathbb{C}^{4 m}: z_{k}=x_{k}+i y_{k}, y_{k} \in V_{+}, k=1, \ldots, m\right\}
$$

и

$$
T_{m}^{-}=\left\{z \in \mathbb{C}^{4 m}: z_{k}=x_{k}-i y_{k}, y_{k} \in V_{+}, k=1, \ldots, m\right\}
$$


являются преобразованиями Фурье-Лапласа двух обобщенных функций умеренного роста на $\mathbb{R}^{4 m}, \tilde{f}^{+}\left(x_{1}, \ldots, x_{m}\right)$ и $\tilde{f}^{-}\left(x_{1}, \ldots, x_{m}\right)$ соответственно. Пусть носители обобщенных функций $\tilde{f}^{+}\left(x_{1}, \ldots, x_{m}\right)$ и $\tilde{f}^{-}\left(-x_{1}, \ldots,-x_{m}\right)$ лежат в $\bar{V}_{+}^{\times m}$. Тогда для всех $z \in T_{m}^{\prime}$ и $A, B \in S L(2, \mathbb{C})$ справедлива следующая формула:

$$
f\left(A \tilde{z}_{1} B, \ldots, A \tilde{z}_{m} B\right)=\sum_{l, i=0,1 / 2, \ldots, L} \sum_{k, n=-l}^{l} \sum_{\dot{k}, \dot{n}=-i}^{i} t_{n k}^{l}(A) t_{\dot{n} \dot{k}}^{i}(B) F_{k, n, \dot{k}, \dot{n}}^{l, i}\left(\tilde{z}_{1}, \ldots, \tilde{z}_{m}\right) .
$$

Здесь матричные элементы $F_{k, n, \dot{k}, \dot{n}}^{l, i}\left(\tilde{z}_{1}, \ldots, \tilde{z}_{m}\right)$ являются голоморфными функциями от $z$ в области $T_{m}^{\prime}$ и имеют те же свойства, что предполагаются для функции $f\left(z_{1}, \ldots, z_{m}\right)$.

Частный случай $(m=1)$ этой теоремы был доказан Боголюбовым и Владимировым [13], [14]. Общий случай этой теоремы был доказан в работах [8], [15] с помощью гипотезы о $m$-точечной расширенной трубе. Гипотеза о $m$-точечной расширенной трубе была доказана в работе [16].

Рассмотрим бесконечный ряд

$$
\int d^{4} x F^{+}(x) \phi(x)=\sum_{l \in \mathbb{Z}_{+} / 2} \sum_{m, \dot{m}=-l}^{l} \int d^{4} x f_{m, \dot{m}}^{l}((x, x)) \theta\left(x^{0}\right)\left(\partial_{x}, \partial_{x}\right)^{q} t_{m \dot{m}}^{l}\left(-\tilde{\partial}_{x}\right) \phi(x),
$$

где непрерывные функции $f_{m, \dot{m}}^{l}(s)$ с носителем на положительной полуоси полиномиально ограничены. Предположим, что этот ряд абсолютно сходится для любой пробной функции $\phi(x) \in S\left(\mathbb{R}^{4}\right)$. Следовательно, обобщенная функция умеренного роста (2.82) имеет носитель в замкнутом верхнем световом конусе $\bar{V}_{+}$. Можно вычислить преобразование Фурье-Лапласа обобщенной функции (2.82):

$$
\begin{gathered}
\int d^{4} x F^{+}(x) e^{i(z, x)}=\sum_{l \in \mathbb{Z}_{+} / 2} \sum_{m, \dot{m}=-l,-l+1, \ldots, l-1, l} \int_{0}^{\infty} s d s f_{m, \dot{m}}^{l}(s) \times \\
\times 2 \pi(-(z, z))^{q} t_{m \dot{m}}^{l}(-i \tilde{z})(-s(z, z))^{-1 / 2} K_{1}\left((-s(z, z))^{1 / 2}\right) .
\end{gathered}
$$

Этот ряд сходится для $z \in T_{1}^{+}$, следовательно, функция, заданная равенством (2.83), является голоморфной по $z$ в трубе $T_{1}^{+}$. Любой член ряда (2.83) имеет аналитическое продолжение по $z$ в область $T_{1}^{\prime}$. Пусть этот ряд сходится для $z \in T_{1}^{\prime}$, тогда функция (2.83) имеет аналитическое продолжение по $z$ в область $T_{1}^{\prime}$.

Поскольку бесконечный ряд (2.82) абсолютно сходится для любой пробной функции $\phi(x) \in S\left(\mathbb{R}^{4}\right)$, бесконечный ряд

$$
\int d^{4} x F^{-}(x) \phi(x)=\sum_{l \in \mathbb{Z}_{+} / 2} \sum_{m, \dot{m}=-l}^{l} \int d^{4} x f_{m, \dot{m}}^{l}((x, x)) \theta\left(-x^{0}\right)\left(\partial_{x}, \partial_{x}\right)^{q} t_{m \dot{m}}^{l}\left(-\tilde{\partial}_{x}\right) \phi(x)
$$

абсолютно сходится для любой пробной функции $\phi(x) \in S\left(\mathbb{R}^{4}\right)$. Следовательно, обобщенная функция умеренного роста $F^{-}(-x)$ имеет носитель в замкнутом верхнем световом конусе $\bar{V}_{+}$. Ограничение функции $(2.83)$ на трубу $T_{1}^{-}$является преобразованием Фурье-Лапласа обобщенной функции (2.84). Теперь из соотношения (2.81) теоремы о конечной ковариантности для случая $m=1$ следует, что 
число ненулевых членов в ряду (2.83) конечно, поэтому число ненулевых членов в рядах (2.82) и (2.84) конечно. Кажется естественным определить ряд (2.82) для функций $\phi(x) \in S\left(\mathbb{R}^{4}\right)$, у которых преобразования Фурье (2.78) имеют компактные носители.

Существует другое доказательство этого результата. Предположим, что бесконечный ряд (2.82) абсолютно сходится для любой пробной функции $\phi(x) \in S\left(\mathbb{R}^{4}\right)$. Тогда обобщенная функция умеренного роста (2.82) имеет носитель в замкнутом верхнем световом конусе $\bar{V}_{+}$. Поскольку бесконечный ряд (2.82) абсолютно сходится для любой пробной функции $\phi(x) \in S\left(\mathbb{R}^{4}\right)$, бесконечный ряд (2.84) абсолютно сходится для любой пробной функции $\phi(x) \in S\left(\mathbb{R}^{4}\right)$. Следовательно, обобщенная функция умеренного роста $F^{-}(-x)$ имеет носитель в замкнутом верхнем световом конусе $\bar{V}_{+}$. Использовав ряд (2.83), можно доказать, что преобразование Фурье разности обобщенных функций (2.82) и (2.84) равно нулю для любого пространственноподобного аргумента. Теперь из представления Йоста-Лемана-Дайсона следует, что число ненулевых членов в рядах (2.82) и (2.84) конечно.

Благодарности. Работа поддержана РФФИ (грант № 07-01-00144) и Программой поддержки ведущих научных школ (грант НШ-672.2006.1).

\section{Список литературы}

[1] A. S. Wightman, L. Gårding, Ark. Fys., 28 (1965), 129-189.

[2] Р. Стритер, А. С. Вайтман, РCT, спин и статистика и все такое, Наука, М., 1966.

[3] Р. Йост, Общая теория квантованных полей, Мир, М., 1967.

[4] Н. Н. Боголюбов, А. А. Логунов, А. И. Оксак, И. Т. Тодоров, Общие принципы квантовой теории поля, Наука, М., 1987.

[5] P. D. Methée, Comment. Math. Helv., 28:1 (1954), 225-269.

[6] L. Gårding, J. L. Lions, Nuovo Cimento, 14:1 (1959), 9-66, Suppl..

[7] Yu. M. Zinoviev, Comm. Math. Phys., 47:1 (1976), 33-42.

[8] J. Bros, H. Epstein, V. Glaser, Comm. Math. Phys., 6:2 (1967), 77-100.

[9] Н.Я. Виленкин, Специальные функции и теория представлений групп, Наука, М., 1965.

[10] В. С. Владимиров, Методы теории функиий многих комплексных переменных, Наука, M., 1964.

[11] В. В. Жаринов, ТМФ, 9:2 (1971), 232-239.

[12] Р. Нарасимхан, Анализ на действительных и комплексных многообразиях, Мир, М., 1971.

[13] Н. Н. Боголюбов, В. С. Владимиров, Науч. докл. высшей школь. Физ.-мат. науки, 3 (1958), 26-35.

[14] Н. Н. Боголюбов, В. С. Владимиров, Науч. докл. высшей школь. Физ.-мат. науки, 2 (1959), 179-179.

[15] Н.Н.Боголюбов, В. С. Владимиров, "Представления $n$-точечных функций", Сборник статей. 1. Посв. акад. И. М. Виноградову к его 80-летию, Труды МИАН, 112, Наука, M., 1971, 5-21.

[16] Ч. Щаньюй, Изв. РАН. Сер. матем., 62:1 (1998), 211-224.

Поступила в редакцию 10.07.2007, после доработки 6.11.2007 